Research Article

\title{
A Novel Blind Deconvolution Method with Adaptive Period Estimation Technique and Its Application to Fault Feature Enhancement of Bearing
}

\author{
Qiuyang Zhou, ${ }^{1}$ Cai Yi $\left(\mathbb{D},{ }^{1}\right.$ Chenguang Huang, ${ }^{2}$ and Jianhui Lin ${ }^{1}{ }^{1}$ \\ ${ }^{1}$ State Key Laboratory of Traction Power, Southwest Jiaotong University, Chengdu 610031, Sichuan, China \\ ${ }^{2}$ Weichai Power Co., Ltd., Weifang, China \\ Correspondence should be addressed to Cai Yi; yicai@swjtu.edu.cn
}

Received 15 July 2021; Accepted 10 November 2021; Published 27 November 2021

Academic Editor: Gang Tang

Copyright (C) 2021 Qiuyang Zhou et al. This is an open access article distributed under the Creative Commons Attribution License, which permits unrestricted use, distribution, and reproduction in any medium, provided the original work is properly cited.

Minimum correlated generalized $L p / L q$ deconvolution (MCG- $L p / L q-\mathrm{D}$ ) is an important tool to detect periodic impulses in vibration mixture. It is proved to be a more stable technique than maximum correlated kurtosis deconvolution (MCKD) to recover the fault impulse under strong noise conditions. However, MCG- $L p / L q$-D still has limitations. One of the necessary conditions for the success of MCG- $L p / L q-D$ is to provide a precise period of fault. An imprecise prior period will lead to performance degradation or even failure of the method. Therefore, in this paper, a MCG- $L p / L q-\mathrm{D}$ with adaptive fault period estimation capability is proposed, adaptive minimum correlated generalized $L p / L q$ deconvolution (AMCG- $L p / L q-D$ ). The proposed method uses the autocorrelation function of envelope signal to estimate the fault period adaptively in each iteration and then takes the estimated period as the input parameter of MCG- $L p / L q-D$ for the next iteration optimization. The proposed method does not require precise prior fault period input, which greatly improves the fault recovery accuracy and application range of MCG- $L p / L q$-D. Eventually, simulated and experimental data verify the effectiveness and superiority of AMCG- $L p / L q$-D.

\section{Introduction}

Fault diagnosis of rotating machinery is very important for making equipment maintenance plan, preventing equipment damage, and ensuring operation safety. Failure of rotating machinery might lead to serious equipment damage and safety accidents. Many advanced fault detection methods have been proposed and applied in engineering practice. These methods can be divided into several categories, including spectral kurtosis [1-3], sparse representation [4-6], cyclostationarity methods [7-10], signal mode decomposition methods [11-13], deep learning $[14,15]$, and blind deconvolution (BD) [16-19]. BD methods can recover fault impulse adaptively from noise observation, which has been developed and promoted in fault diagnosis.

The essence of $\mathrm{BD}$ is to adaptively construct an inverse filter to eliminate the influence of the transmission path on the collected vibration signal [20]. According to whether the prior knowledge of the period is used, $\mathrm{BD}$ can be divided into nonperiod dependent $\mathrm{BD}$ and period dependent $\mathrm{BD}$. Minimum entropy deconvolution (MED) is a representative example of nonperiod dependent $\mathrm{BD}[16]$ and has been applied to bearing fault diagnosis by Sawalhi et al. [21]. It can recover the fault impulse in the original vibration signal by designing a filter based on the maximum kurtosis of the filtered signal. However, many researches show that MED is sensitive to large peaks in signals $[18,22]$ and not robust enough to noise $[23,24]$, leading to the fact that MED might not be able to recover repetitive fault-related impulses. Aiming at the deficiency of MED, Jia et al. [18] proposed sparse filtering (SF) with the generalized $L p / L q(\mathrm{G}-L p / L q)$ norm with $p<q$. Compared with kurtosis, the G- $L p / L q$ norm is less sensitive to large peaks in signals $[22,25]$, which makes SF based on the G- $L p / L q$ norm minimization more robust to large impulses and noise than MED. However, when there is strong background noise in the vibration signal, SF usually needs to use a smaller $p$ value to improve the robustness of the method. The use of smaller $p$ values will 
reduce the sensing ability of the G- $L p / L q$ norm to fault impulses [22, 25], resulting in the failure of SF to recover any fault features. In order to overcome the defects of MED and SF simultaneously, BD using the fault impact periodic nature (period dependent BD) is developed.

Maximum correlated kurtosis deconvolution (MCKD) [24] is a typical period dependent BD. Compared with MED, MCKD uses prior knowledge of fault impulses period to enhance the periodic fault impulses submerged in noise [24]. MCKD can adaptively design the target filter by maximizing the correlated kurtosis [24] (CK) of the filtered signal. CK can not only emphasize the high kurtosis of signal, but also highlight the periodic impulse. MCKD has stronger recovery performance of the fault impulse than MED. Subsequently, multipoint optimal minimum entropy deconvolution adjusted (MOMEDA) [23] based on multi-D-norm maximization is proposed to directly solve the optimal filter. However, the case studies in $[26,27]$ prove that both MCKD and MOMEDA are not robust enough to process strong noise signals, while BD based on the correlated generalized $L p / L q(\mathrm{CG}-L p / L q)$ norm minimization (minimum correlated generalized $L p / L q$ deconvolution, MCG- $L p / L q-D)$ shows better robustness to noise. This is because the CG- $L p / L q$ norm analyzes the correlation function of the filtered signal rather than the filtered signal itself [26], which can be regarded as the extension of the $\mathrm{G}-L p / L q$ norm in the signal correlation domain. Therefore, CG- $L p / L q$ norm is a low-order statistic and also emphasizes the periodicity of the impulse train, which makes MCG- $L p / L q-\mathrm{D}$ more robust to random large peaks and noise in the signal, thus making it more suitable for processing strong noise signals. However, MCG- $L p / L q-\mathrm{D}$ is very demanding for the prior fault period input precision. In engineering practice, due to the influence of both rotation speed fluctuation and measurement error $[28,29]$, the precise fault period is usually hard to be obtained through theoretical calculation. This greatly limits the application of MCG- $L p / L q-\mathrm{D}$ in engineering practice. The purpose of this study is to overcome the limitation of the dependence on the precise fault period of MCG-Lp/Lq-D.

Recently, in order to overcome the limitation of the dependence on fault period for MCKD, Miao et al. proposed improved MCKD (IMCKD) [30]. IMCKD uses the autocorrelation function of the envelope signal for filtered signal (adaptive period estimation method, APE [31-33]) to estimate the fault period of signal. It solves the problem that MCKD is dependent on prior period, thus improving MCKD to be a nonperiod dependent method. Inspired by IMCKD, APE can be considered to be introduced into MCG- $L p / L q-\mathrm{D}$ to construct to overcome the second limitation of MCG-Lp/Lq-D. However, when the SNR is very low, especially when there are concurrent faults in the vibration signal [34], APE might fail to detect the real fault period, which will cause MCG- $L p / L q-\mathrm{D}$ to converge to the incorrect result $[34,35]$. Actually, in most engineering practice, the fault period can be obtained by theoretical calculation according to the rotation speed and the component geometry parameter $[6,36,37]$. Although the rotation speed fluctuation and the measurement error of component geometry parameter might cause the difference between the theoretical calculation value and the real value of the fault impulse period, it is an approximate estimation of the real fault period. Therefore, when APE fails under strong noise, the theoretical calculation period will be a good input of period parameters for MCG- $L p /$ $L q$-D. In other words, this theoretical calculation period can be used to guide APE to track the real fault period to ensure the precision of the input period used by MCG- $L p / L q-\mathrm{D}$.

According to the above discussion, a new deconvolution method, the adaptive MCG- $L p / L q-\mathrm{D}$ (AMCG- $L p / L q-\mathrm{D}$ ), is proposed in this paper. AMCG- $L p / L q-\mathrm{D}$ uses the improved APE (adaptive period estimation with constraint, APEC) method to estimate the period parameters needed by MCG$L p / L q-D$. The principle of APEC is as follows. Firstly, according to the theoretical calculation period, the APEC constructs the constraint range of the period estimation which contains the real fault period. Then, the APEC is used to estimate the period within this constraint range, and the estimated period is regarded as the fault period used in this iteration. If the APEC still fails within this constraint range, the theoretical calculation period is regarded as the fault period used in this iteration. Firstly, when there is an error between the input period and the real fault period, the use of APEC can make MCG-Lp/Lq-D no longer iterate with an imprecise period, which is beneficial to improve the performance of fault recovery. Because in engineering practice, the prior fault period obtained through theoretical calculation is often not precise, the use of APEC ensures that MCG- $L p / L q-D$ can use precise fault period for iteration, thus expanding the practicality of MCG- $L p / L q-D$. Additionally, because the APE technique used in the IMCKD is completely adaptive, when there is strong noise interference in the signal, the estimated period might differ greatly from the real fault period. By contrast, the APEC technique can make the proposed method avoid using the period parameters which are greatly different from the real fault period during iteration. This is beneficial to improve the computational efficiency of the proposed method. Furthermore, AMCG- $L p / L q$-D inherits the original advantages of MCG- $L p / L q-\mathrm{D}$, including excellent robustness to noise and concurrent fault diagnosis ability.

The rest of this paper is arranged as follows. Section 2 reviews the basic theory of MCG- $L p / L q-D$. In Section 3, the limitations of APE are discussed. APEC is proposed, and based on this, AMCG- $L p / L q-\mathrm{D}$ is proposed. In Sections 4 and 5, the performances of AMCG-Lp/Lq-D in the processing of vibration mixtures separate containing single fault signal and concurrent fault signal through simulated and experimental data. Finally, the conclusions are in Section 6.

\section{Theoretical Review}

MCG- $L p / L q-\mathrm{D}$ is a deconvolution method based on the CG$L p / L q$ norm. The CG- $L p / L q$ norm is defined as follows:

$$
J_{p, q}(\operatorname{Corr}(y, T))=\left(\frac{\left(\sum_{n=1}^{N}\left(y_{n-T} y_{n+T}\right)^{p}\right)^{1 / p}}{\left(\sum_{n=1}^{N}\left(y_{n-T} y_{n+T}\right)^{q}\right)^{1 / q}}\right)^{p}, \quad q>p>0,
$$

where $\operatorname{Corr}(\mathbf{y}, T)$ is the correlation function related to filtered signals $\mathbf{y}_{1}=\mathbf{y}(1: N-T)$ and $\mathbf{y}_{2}=\mathbf{y}(T+1: N)$. $\mathbf{v}$ is 
used to denote the correlation function, $\mathbf{v}=\operatorname{Corr}(\mathbf{y}, T)=\mathbf{y}_{1} \mathbf{y}_{2} . T$ is the precise period (in sample) of the fault impact in the vibration signal. It can be calculated by $T=F s / F_{d}$, where $F s$ is the sampling frequency, and $F_{d}$ is the fault feature frequency. The definition of MCG- $L p / L q-\mathrm{D}$ is represented as

$$
\begin{aligned}
\min _{f} J_{p, q}(\mathbf{v}) & =\left(\frac{\left\|\mathbf{y}_{1} \mathbf{y}_{2}\right\|_{p}}{\left\|\mathbf{y}_{1} \mathbf{y}_{2}\right\|_{q}}\right)^{p} \\
& =\left(\frac{\left(\sum_{n=1}^{N}\left(y_{n-T} y_{n+T}\right)^{p}\right)^{1 / p}}{\left(\sum_{n=1}^{N}\left(y_{n-T} y_{n+T}\right)^{q}\right)^{1 / q}}\right)^{p},
\end{aligned}
$$

where $\mathbf{f} \in \mathbb{R}^{L}$ is the desired filter. The output signal after deconvolution is obtained by $\mathbf{y}=\mathbf{x} * \mathbf{f},\|\mathbf{f}\|_{2}=1$, and $\mathbf{x} \in \mathbb{R}^{N}$ is the input vibration mixture signal. For the convenience of numerical calculation, Hankel matrix $\mathbf{H} \in \mathbb{R}^{(N-L+1) \times L}$ is constructed based on $\mathbf{x}$. Therefore, the output result after deconvolution becomes

$$
\mathbf{y}=\mathbf{H f},\|\mathbf{f}\|_{2}=1,
$$

where $\mathbf{H}$ is defined as

$$
\mathbf{H}=\left[\begin{array}{ccccc}
x_{1} & x_{2} & x_{3} & \cdots & x_{L} \\
x_{2} & x_{3} & x_{4} & \cdots & x_{L+1} \\
x_{3} & x_{4} & x_{5} & \cdots & x_{L+2} \\
\vdots & \vdots & \vdots & \vdots & \vdots \\
x_{N-L+1} & x_{N-L+2} & x_{N-L+3} & \cdots & x_{N}
\end{array}\right]_{N-L+1 \text { by } L} .
$$

At this point, the definition of $\mathbf{v}$ can be expressed as

$$
\begin{aligned}
\mathbf{v} & =\mathbf{y}(1: N-L+1-T) \cdot \mathbf{y}(T+1: N-L+1) \\
& =(\mathbf{H}(1: N-L+1-T,:) \cdot \mathbf{f}) \cdot(\mathbf{H}(T+1: N-L+1,:) \cdot \mathbf{f}),
\end{aligned}
$$

where $\mathbf{H}(1: N-L+1-T,:)$ is the new matrix of the Hankel matrix from the first row to the $N-L+1-T$ th row, represented by $\mathbf{H}_{1} . \mathbf{H}(T+1: N-L+1,:)$ is the new matrix of the Hankel matrix from the $T+1$ th row to the $N-L+1$ th row, represented by $\mathbf{H}_{2}$. The definitions of $\mathbf{H}_{1}$ and $\mathbf{H}_{2}$ are separately shown as follows:

$$
\begin{aligned}
\mathbf{H}_{1} & =\left[\begin{array}{ccccc}
x_{1} & x_{2} & x_{3} & \cdots & x_{L} \\
x_{2} & x_{3} & x_{4} & \cdots & x_{L+1} \\
x_{3} & x_{4} & x_{5} & \cdots & x_{L+2} \\
\vdots & \vdots & \vdots & \vdots & \vdots \\
x_{N-L+1-T} & x_{N-L+2-T} & x_{N-L+3-T} & \cdots & x_{N-T}
\end{array}\right], \\
\mathbf{H}_{2} & =\left[\begin{array}{ccccc}
x_{T+1} & x_{T+2} & x_{T+3} & \cdots & x_{T+L} \\
x_{T+2} & x_{T+3} & x_{T+4} & \cdots & x_{T+L+1} \\
x_{T+3} & x_{T+4} & x_{T+5} & \cdots & x_{T+L+2} \\
\vdots & \vdots & \vdots & \vdots & \vdots \\
x_{N-L+1} & x_{N-L+2} & x_{N-L+3} & \cdots & x_{N}
\end{array}\right]
\end{aligned}
$$

Due to the fact that the minimization of $J_{p, q}(\mathbf{v})$ is still a nonsmoothness problem, $\mathbf{c}=\left(\mathbf{v}^{2}+\varepsilon\right)^{1 / 2}$ is used to replace $\mathbf{v}$ [26]. According to equations (4)-(6), the minimization problem in equation (2) can be described by the following equation:

$$
\begin{aligned}
\min _{\mathbf{f}} J_{p, q}(\mathbf{v}) & =\left(\frac{\|\mathbf{c}\|_{p}}{\|\mathbf{c}\|_{q}}\right)^{p}, \quad p<q, \\
\text { s.t. } \mathbf{v} & =\mathbf{y}_{1} \mathbf{y}_{2}, \\
\mathbf{y}_{1} & =\mathbf{H}_{1} \mathbf{f}, \\
\mathbf{y}_{2} & =\mathbf{H}_{2} \mathbf{f}, \\
\|\mathbf{f}\|_{2} & =1 .
\end{aligned}
$$

The optimization problem in (7) can be solved by the gradient descent algorithm [38]. The gradient can be expressed as

$$
\begin{aligned}
\frac{\mathrm{d} J_{p, q}}{\mathrm{~d} \mathbf{f}}= & p \cdot\left(\frac{\|\mathbf{c}\|_{p}}{\|\mathbf{c}\|_{q}}\right)^{(p-1)} \cdot\left(\frac{\mathbf{v}}{\mathbf{c}}\right) \cdot\left(\mathbf{y}_{2} \cdot \mathbf{H}_{1}+\mathbf{y}_{1} \cdot \mathbf{H}_{2}\right) \\
& \cdot\left(\frac{\left(\|\mathbf{c}\|_{p}\right)^{(1-p)} \cdot \mathbf{c}^{(p-1)} \cdot\|\boldsymbol{c}\|_{q}-\left(\|\mathbf{c}\|_{q}\right)^{(1-q)} \cdot \mathbf{c}^{(q-1)} \cdot\|\mathbf{c}\|_{p}}{\left(\|\boldsymbol{c}\|_{q}\right)^{2}}\right) .
\end{aligned}
$$

The specific steps of MCG-Lp/Lq-D are as follows:

Step 1: Input the precise fault period $T$, convert the input signal $\mathbf{x}$ into Hankel $\mathbf{H}$, and obtain the Hankel matrixes $\mathbf{H}_{1}$ and $\mathbf{H}_{2}$.

Step 2: Initialize the filter coefficients, $\mathbf{f}_{0}=[0,1,0, \ldots, 0]$.

Step 3: Obtain $J_{p, q}(\mathbf{v})$ through forward propagation; calculate $\mathrm{d} J_{p, q} / \mathrm{df}$ based on equation (8).

Step 4: Update filter coefficients by gradient descent algorithm.

Step 5: Repeat Steps 3 and 4 until the stop criteria are fulfilled.

\section{Proposed Deconvolution Technique}

3.1. Adaptive Period Estimation Method. Since the fault signal of rotating machinery is usually a nonstationary amplitude modulation signal, the envelope demodulation signal is easier to judge the fault information contained in the signal than the original signal [39]. According to the definition of autocorrelation function, the position corresponding to the maximum value of the autocorrelation function for the envelope signal is the best estimation of the period for fault signal. Theoretically, the periodicity of the signal can be accurately detected by using envelope signal. The autocorrelation function of the envelope is defined as follows:

$$
E_{x}(t)=|x(t)+j(\operatorname{Hilbert}(x(t)))|,
$$


where $E_{x}(t)$ is the envelope signal of time series $x(t)$. Hilbert $(\cdot)$ represents the Hilbert transform. $j$ is the unit for the imaginary part.

$$
A_{x}(\tau)=\int E_{x}(t) E_{x}(t+\tau) \mathrm{d} t
$$

where $A_{x}(\tau)$ is the envelope autocorrelation function with respect to lag $\tau$.

Figure 1 illustrates the envelope autocorrelation function of a periodic fault impulse whose real fault period is $T_{r}$. According to the definition of envelope autocorrelation spectrum, when $\tau \approx T_{r}$, the envelope autocorrelation function of the fault signal reaches its maximum value (except zero point) [40]. Therefore, the lag $\tau$ corresponding to the maximum value of $A_{x}(\tau, \tau \neq 0)$ is the period (in sample) of fault impulse. In order to distinguish the real period from the estimation period, the estimation period is denoted as $T_{e}$.

A simulation signal with $\mathrm{SNR}=-2 \mathrm{~dB}$ is used to illustrate the effectiveness of APE, where the sampling frequency of signal $F s$ is $10 \mathrm{kHz}$, the fault feature frequency $F_{d}$ is $103 \mathrm{~Hz}$, and corresponding fault period is equal to 98 . The simulation signal is plotted in Figure 2(a). Because the simulation signal has a high SNR, its impulse features can be clearly observed. Figure 2(b) illustrates the envelope autocorrelation function of simulation signal, where $T_{e}=98$. The period estimated in Figure 2(b) is the same as the real fault period. This shows that APE can accurately estimate the fault period of simulation signal when $\mathrm{SNR}=-2 \mathrm{~dB}$.

When $\mathrm{SNR}=-8 \mathrm{~dB}$, the waveform of the simulation signal is shown in Figure 3(a). It can be seen from Figure 3(a) that the fault impulse is disturbed by noise, and its impulse is hard to be observed. Its envelope autocorrelation function is presented in Figure 3(b), where the period detected by APE is $T_{e}=4$. This lag corresponds to a global maximum point, which suggests that APE might not be able to correctly detect the real fault period under strong noise interference. However, by careful observation of Figure 3(b), it can be found that, near the locations marked by green line and black dot, a local maximum of envelope autocorrelation function can be observed. Its corresponding lag is exactly 98 , which suggests that a fully adaptive period estimation based on APE might fail. However, it is possible to obtain the real fault period if APE is used in a local range containing the real fault period.

When SNR $=-13 \mathrm{~dB}$, the fault impulse is completely submerged by strong noise, and it can hardly be observed, as shown in Figure 3(c). Its envelope autocorrelation function is presented in Figure 3(d), where the period detected by APE is $T_{e}=4$ which is greatly different from the real fault period. Additionally, a local maximum is not found in local range near 98 . This indicates that the real fault period cannot be detected correctly by completely relying on APE for period detection under the condition of low SNR [41].

In summary, when the SNR is high, APE can correctly estimate the period of the signal. As the noise increases, the APE will gradually fail, but APE still has possibility to detect the real fault period in a local range containing the real fault period. When the SNR is very low, APE cannot detect the

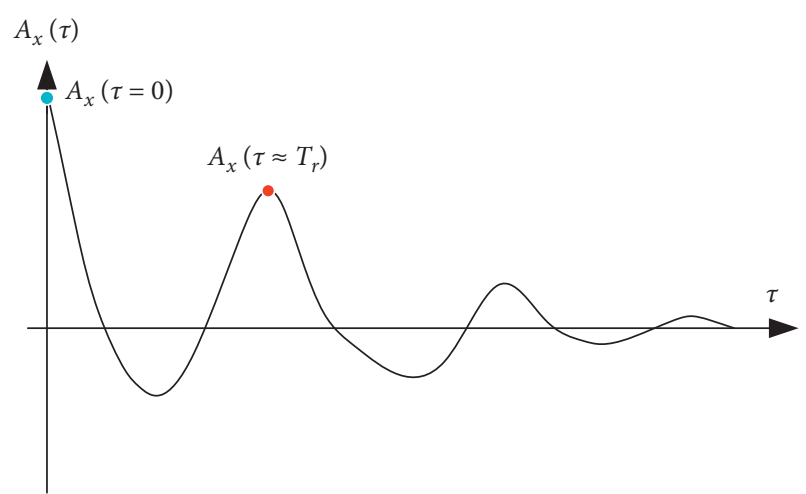

FIGURE 1: Envelope autocorrelation spectrum of signal.

real fault period even in the local range containing the real fault period. The latter two are the two defects of APE in processing the signals with strong noise, which might affect the performance of BD method based on APE (such as IMCKD). In order to overcome the above defects, this paper proposes a period detection method, APE with constraint, APEC, which uses the prior period to guide APE to perform period selection.

3.2. Adaptive Period Estimation with Constraint. For the first defect, when the SNR of signal is relatively low, APE is interfered by other local maximums and cannot detect the real fault period. However, it can be found from Figure 3(b) that the real fault period is the maximum within a local range of the envelope autocorrelation function. Therefore, it can be considered to detect the real fault period in this local range to avoid other interference maximum points, such as 4 in Figure 3(b). Based on this idea, a constraint range can be constructed based on the real fault period and its corresponding random fluctuation $[28,29]$. The real fault period is always hard to be obtained in engineering practices. The theoretical calculation period is usually used to replace the real fault period [36]. However, due to the influence of random fluctuation, there is a difference between the theoretical calculation period and the real fault period, with the range within $2 \%[28,29]$. Therefore, the theoretical calculation period $T$ and the difference caused by random fluctuation can be introduced to construct the constraint range containing the real fault period in this paper. This constraint range can be used by APE to estimate the period, avoiding interference from other local maximums. The steps of constraint construction are as follows: Firstly, the theoretical calculation period $T$ is calculated based on $[6,36,37]$. Secondly, based on the random fluctuation range in $[28,29]$, in order to extend the application range of the proposed method, 5\% random fluctuation is used in this paper to construct this constraint, $[T-5 \% T, T+5 \% T]$, named $T_{C}$. Thirdly, APE is used to estimate the period within the constraint range. For the second defect, when the SNR of signal is extremely low, APE cannot find the local maximum value within the constraint range containing the real fault period, as shown in Figure 3(d). The value of theoretical calculation period $T$ 


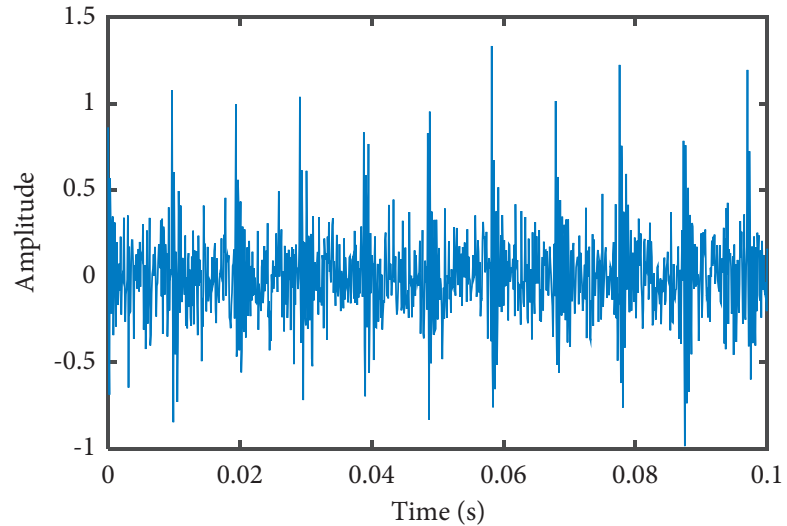

(a)

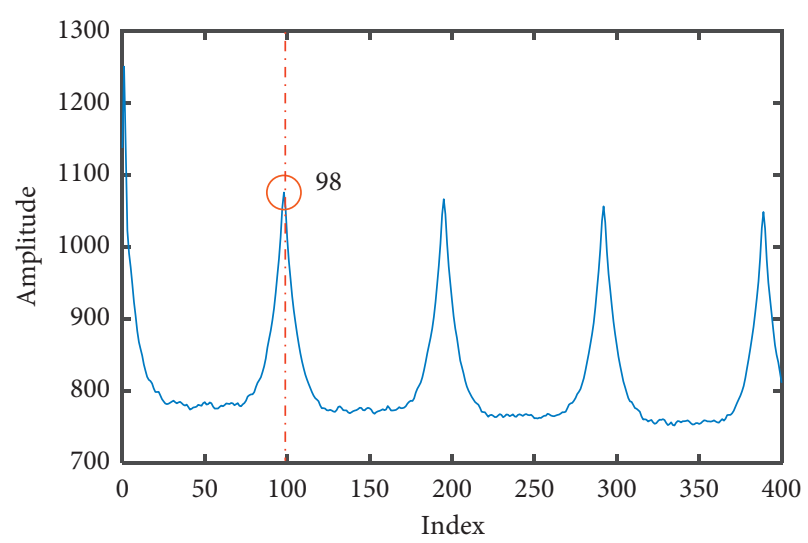

(b)

FIGURE 2: Simulation signal with SNR $=-2 \mathrm{~dB}$ : (a) waveform, (b) envelope autocorrelation spectrum.

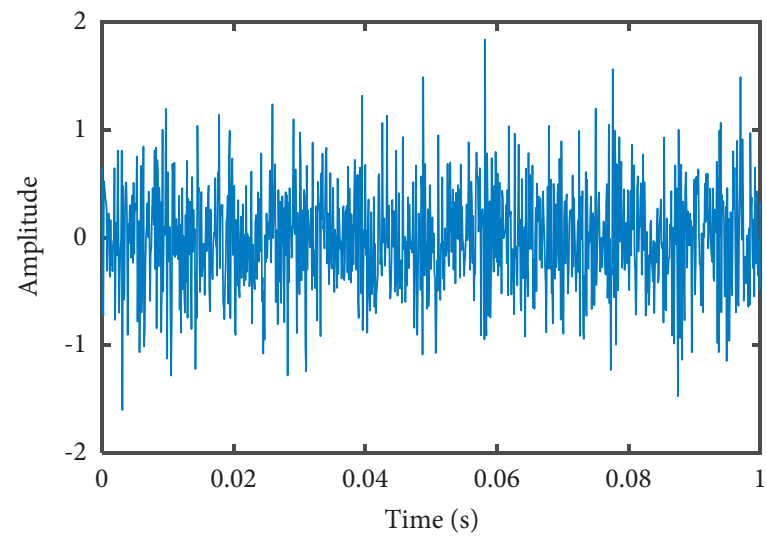

(a)

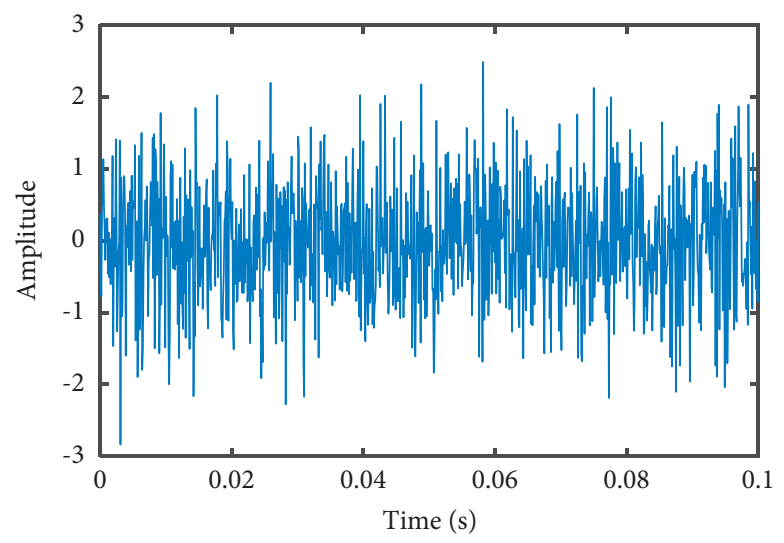

(c)

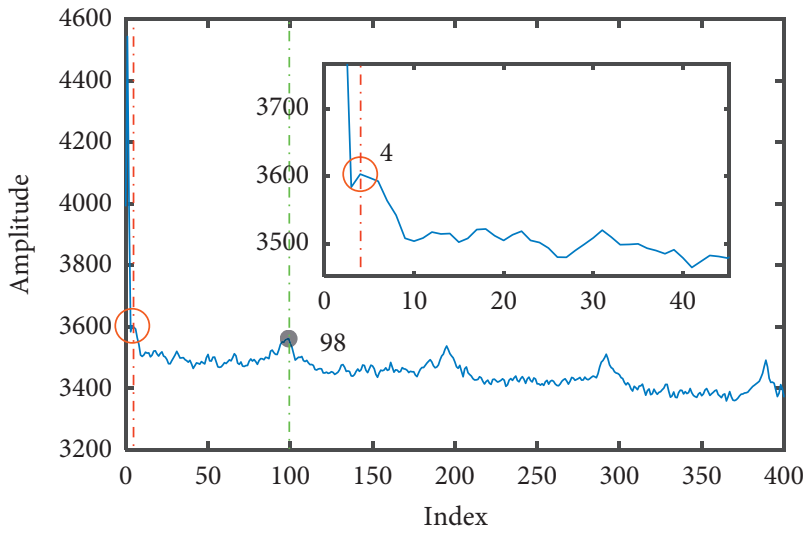

(b)

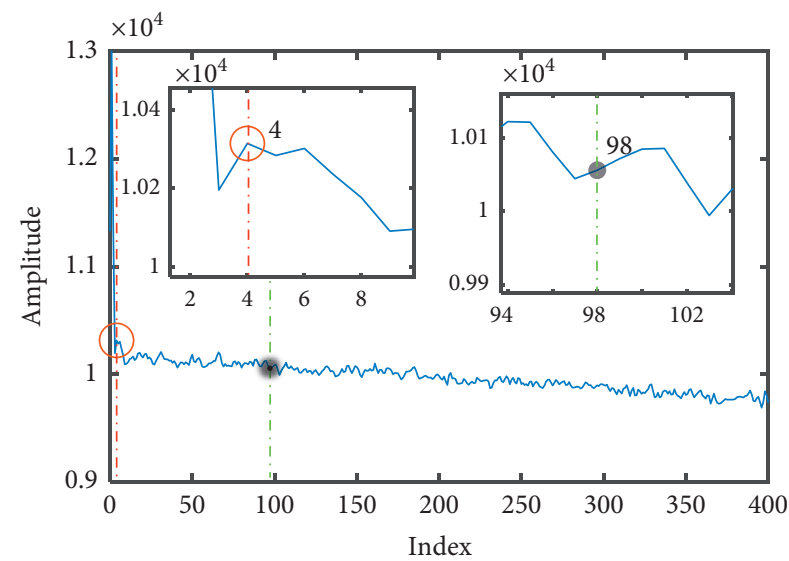

(d)

FIGURE 3: Simulation signals with $\mathrm{SNR}=-8 \mathrm{~dB}$ and $\mathrm{SNR}=-13 \mathrm{~dB}$, respectively: (a) waveform with $\mathrm{SNR}=-8 \mathrm{~dB}$, (b) envelope autocorrelation spectrum with $\mathrm{SNR}=-8 \mathrm{~dB}$, (c) waveform with $\mathrm{SNR}=-13 \mathrm{~dB}$, (d) envelope autocorrelation spectrum with $\mathrm{SNR}=-13 \mathrm{~dB}$.

is assigned to the estimation period $T_{e}$ to ensure that APE can still give a period approximate to the real fault period. By improving the above two defects, the proposed APEC can ensure that the estimation period $T_{e}$ will not deviate from the real fault period $T_{r}$ to a large range even in the case of the failure of APE.
3.3. Adaptive Minimum Correlated Generalized $L p / L q$ Deconvolution. It can be known from IMCKD that the introduction of period estimation technology can improve the performance of MCKD. Inspired by IMCKD, this paper introduces APEC into MCG- $L p / L q-\mathrm{D}$, thus proposing AMCG- $L p / L q$-D. The proposed method is a novel fault 
detection technique, which can adaptively find the real fault period in each iteration by APEC to realize deconvolution algorithm. The flowchart of the proposed method is given in Figure 4. The specific implementation steps of AMCG-Lp/ $L q-\mathrm{D}$ are as follows:

Step 1: Input the theoretical calculation period $T$, select the maximum count of iteration $n$ and the length of filter $L$, and initialize the filter coefficients of AMCG$L p / L q-\mathrm{D}, \mathbf{f}_{0}=[0,1,0, \ldots, 0]$. The count of iteration $i=1$.

Step 2: Construct the constraint $T_{C}$ containing the real fault period to obtain APEC, according to $T$.

Step 3: Obtain the estimation period $T_{e}$ by APEC. Format the Hankel matrix $\mathbf{H}$, according to $T_{e}$, and obtain the Hankel matrixes $\mathbf{H}_{1}$ and $\mathbf{H}_{2}$.

Step 4: Obtain $J_{p, q}(\operatorname{Corr}(\mathbf{x}, T))$ by forward propagation; subsequently calculate the gradient term $\mathrm{d} J_{p, q} / \mathrm{df}$ based on equation (8).

Step 5: Update the filter coefficients $\mathbf{f}$ by gradient descent algorithm.

Step 6: Calculate the output signal filtered by the updated filter, $\mathbf{y}^{(i)}=\mathbf{x}^{T} * \mathbf{f}$.

Step 7: Repeat Steps 3 to 6 until the maximum number of iterations is reached.

Considering that the length of filter will also affect the performance of the proposed method, a recommended selection strategy for filter length is proposed to ensure that the filter can completely cover the whole fault resonance frequency band. Equation (11) should be satisfied when selecting the filter length.

$$
L>2 F s / f_{R S},
$$

where $f_{R S}$ is the resonance frequency. Due to the iteration process of the proposed method, too long filter length will significantly increase the computational complexity, whereas too short filter length will cause performance deterioration of the proposed method. Therefore, in order to avoid the above two extreme results, a tradeoff must be made when choosing the filter length. It should be noted that in order to ensure the fairness and effectiveness of the proposed method compared with other methods, in the subsequent simulation analysis section and experimental analysis section, all the $\mathrm{BD}$ methods used for processing vibration signals use filter length of 100 , and the maximum number of iterations is 400 . Additionally, $p=1$ and $q=2$ are used by both AMCG- $L p / L q-D$ and MCG- $L p / L q-D$. The sampling frequencies of all measured signals are all $10 \mathrm{kHz}$; the sampling time is $1 \mathrm{~s}$.

\section{Simulation Case Studies}

Two bearing simulation signals that separately contain single fault and concurrent fault are used to test the performance of the proposed method. As a comparison, IMCKD, MCKD, and MCG- $L p / L q-\mathrm{D}$ are also used to process these simulated vibrations.
4.1. Single-Fault Simulation Signal Test. According to equation (11) [24, 42] a set of impacts is designed:

$$
\begin{aligned}
\mathbf{S}(t)= & \sum_{n=1}^{N} A_{n} \mathbf{h}\left(t-n T_{r}-\sum_{i=0}^{n} \tau_{i}\right) \\
& \cdot \cos \left(2 \pi f_{R S}\left(t-n T_{r}-\sum_{i=0}^{n} \tau_{i}\right)\right) \mathrm{e}^{-\beta\left(t-n T_{r}-\sum_{i=0}^{n} \tau_{i}\right)},
\end{aligned}
$$

where $A$ represents the amplitude of the simulated, and $\mathbf{h}(t)$ represents the unit step function. The real fault period is $T_{r}=F s / F_{d} \cdot \tau_{i}$ denotes the effects of random slippage, which usually accounts for $1-2 \%$ of $T_{r}$. $\beta$ denotes the structure damper coefficient. Table 1 shows the parameters of simulation bearing fault signal. A Gaussian noise whose SNR = $-12 \mathrm{~dB}$ is added to the simulation signal $\mathbf{S}(t)$.

The single fault simulation vibration signal is shown in Figure 5(a), and its envelope spectrum is shown in Figure 5(b). The red dotted lines represent harmonics corresponding to $F_{d}=113 \mathrm{~Hz}$.

In order to prove the robustness of the proposed method to the input prior period. Their prior fault feature frequency will be set as $108 \mathrm{~Hz}$ and $116 \mathrm{~Hz}$ that deviate from the real fault feature frequency, and the corresponding fault period will be 92.6 and 86.2, respectively. The deviation fluctuation between these two periods and the real period is within 5\%. MCG- $L p / L q-\mathrm{D}$ and MCKD use the same prior period input as AMCG- $L p / L q-\mathrm{D}$. The signal in Figure 5 is processed by AMCG- $L p / L q-\mathrm{D}, \mathrm{MCG}-L p / L q-$ $\mathrm{D}, \mathrm{MCKD}$, and IMCKD, respectively. The results are shown in Figures 6-8.

When the input fault feature frequency is set to $116 \mathrm{~Hz}$ higher than the real fault feature frequency, it can be observed that only the proposed MCG- $L p / L q-\mathrm{D}$ can detect the fault features of bearing and greatly enhance its amplitude, as shown in Figures 5(b), 6(b), 6(d) and 6(f). The variation curve of the period obtained by APEC during the iteration process is plotted in Figure 9(a). It can be found that APEC converges to $T_{e}=88 \approx 10000 / 113=$ $T_{r}$ at the red dot mark, which indicates that even when the prior period is imprecise, AMCG- $L p / L q-\mathrm{D}$ can adaptively correct the period through APEC and eventually converge to the real period. However, neither MCG-Lp/Lq-D nor MCKD can detect the fault features, which indicates that these two methods have higher requirements on the precision of the prior period. This is also the main limitation of $M C G-L p / L q-D$ and $M C K D$ in engineering practice.

When the input fault feature frequency is set to $108 \mathrm{~Hz}$ lower than the real fault feature frequency, the proposed AMCG- $L p / L q-D$ still has a good fault feature recovery performance by comparing envelope spectra in Figures 5(b), 8(b), 8(d), and 8(f). By observing Figure 9(b), APEC converges from the red dot mark to $T_{e}=89 \approx 10000 / 113=T_{r}$, which again proves the superiority of AMCG-Lp/Lq-D. Although MCG- $L p / L q-\mathrm{D}$ also detects the real fault feature frequency, its recovered feature amplitude is significantly smaller than AMCG-Lp/Lq-D. 


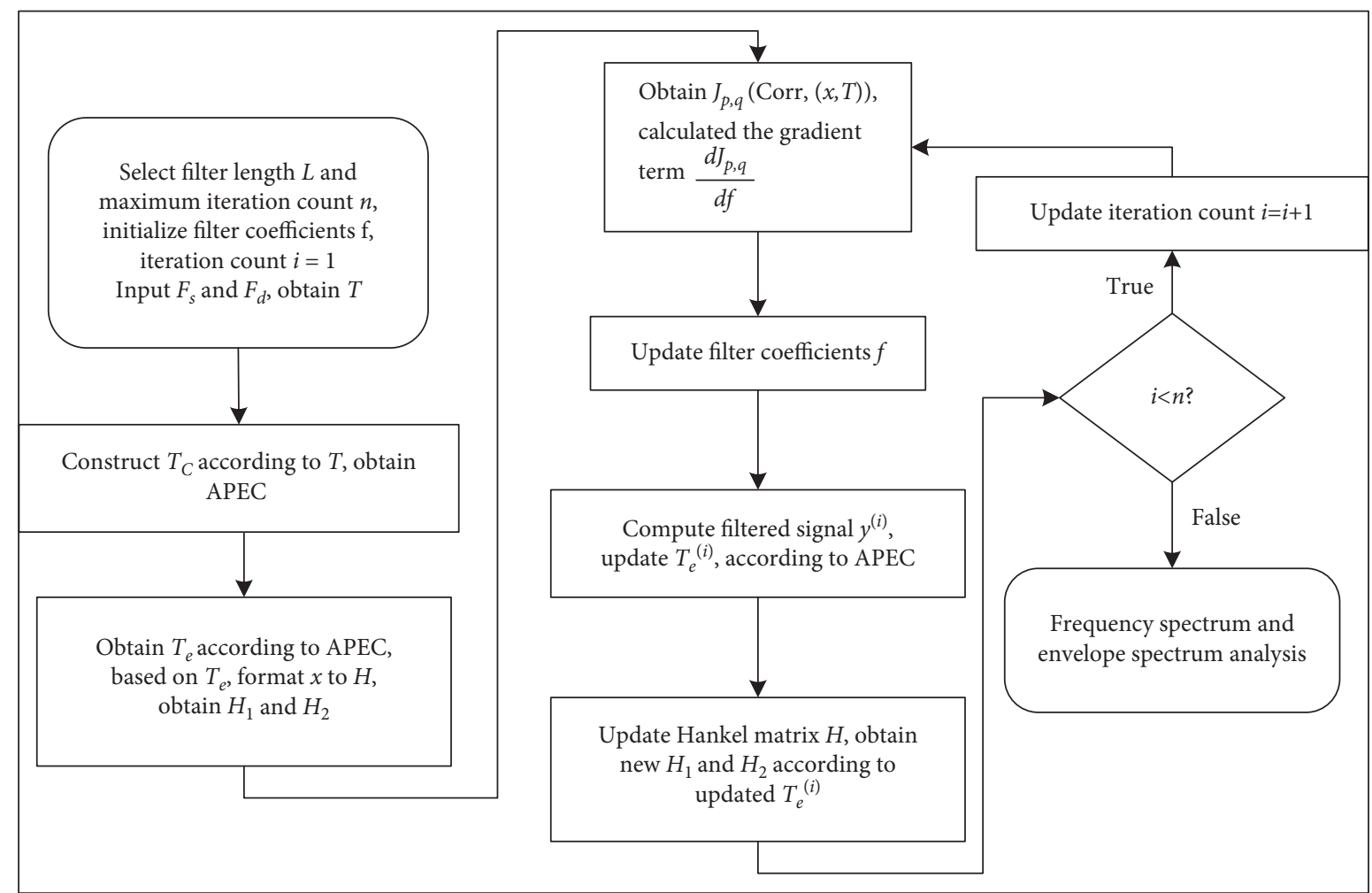

FIGURE 4: Flowchart of AMCG-Lp/Lq-D.

TABLe 1: Parameters of single fault simulation vibration signal.

\begin{tabular}{lcccc}
\hline$A$ & $\beta$ & $f_{R S}(\mathrm{~Hz})$ & $F_{d}(\mathrm{~Hz})$ & $f_{r}(\mathrm{~Hz})$ \\
\hline 1 & 1000 & 3000 & 113 & 10.29 \\
\hline
\end{tabular}

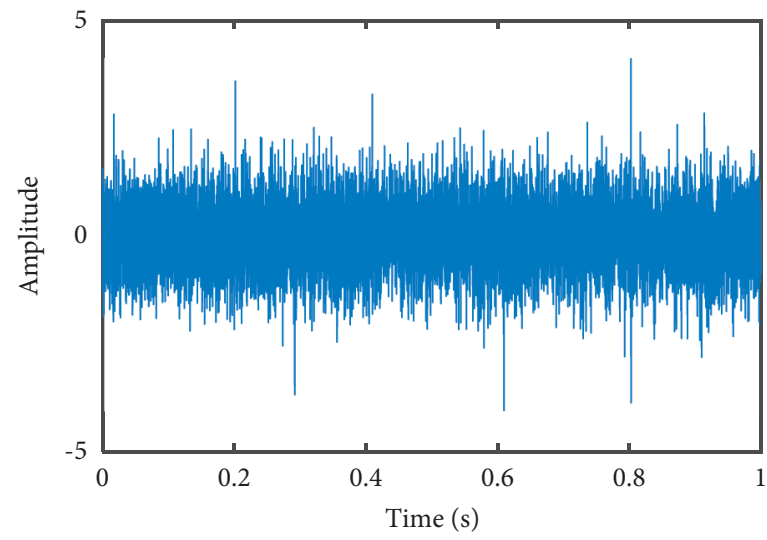

(a)

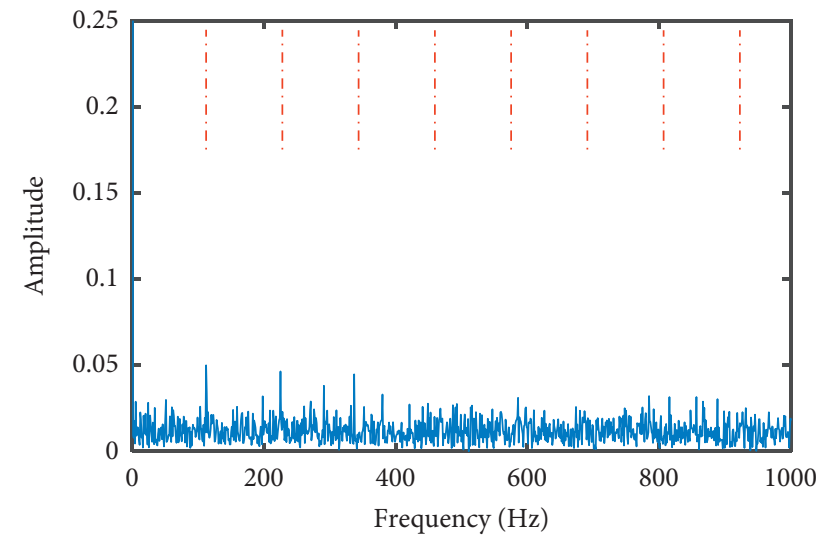

(b)

FIGURE 5: Single fault simulation vibration signal: (a) waveform; (b) envelope spectrum.

By contrast, as shown in Figures 7(a) and 7(b), IMCKD cannot detect the fault features under the condition of strong noise. The variation of the estimation period for IMCKD during the iteration is shown in Figure 9(c). It can be seen that although IMCKD can also update the fault period, it cannot converge to the real fault period. This indicates that APE has a large defect in processing strong noise signals.
In order to fully study the computational complexity of AMCG-Lp/Lq-D, the computational efficiency of the four $\mathrm{BDs}$ is given in Table 2. It is worth noting that the calculation times shown in Table 2 are the average of each method running 30 times. The equipment used is $2.60 \mathrm{GHz}$ Intel Core i7-6700HQ. The software version used is Matlab R2019 B. It can be seen that, due to the increase of APEC 


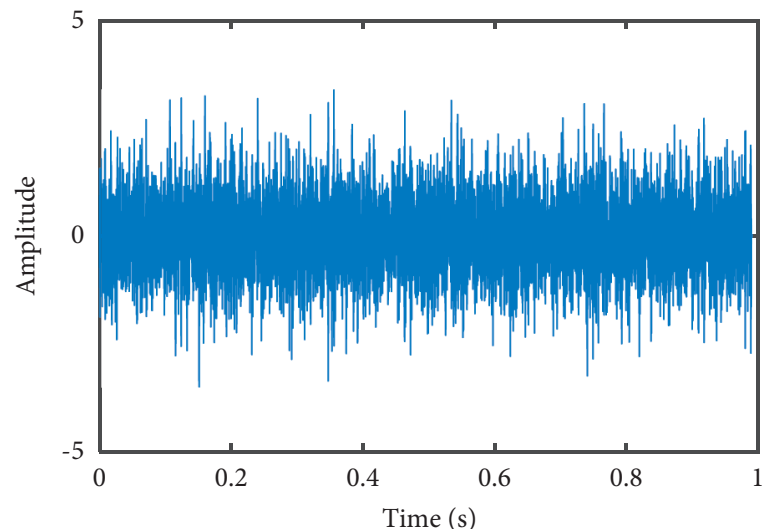

(a)

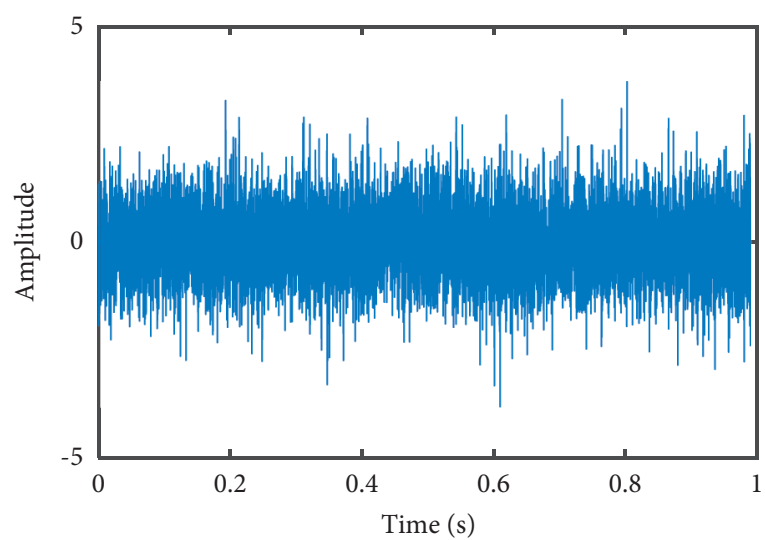

(c)

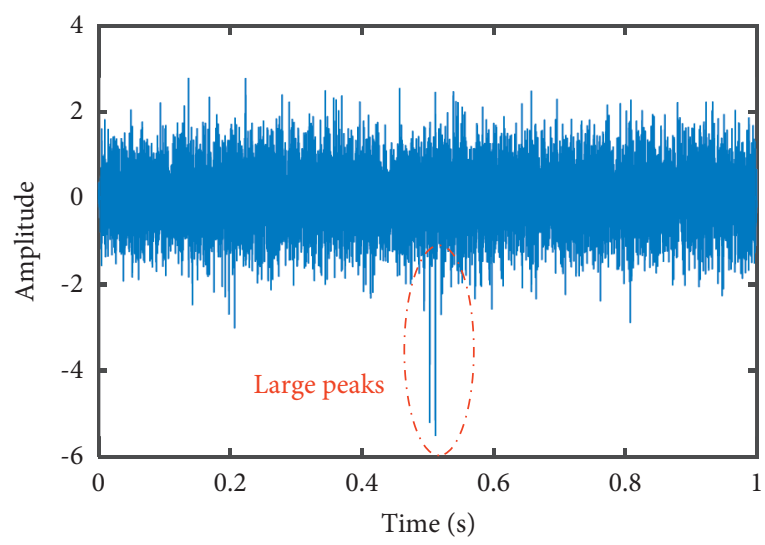

(e)

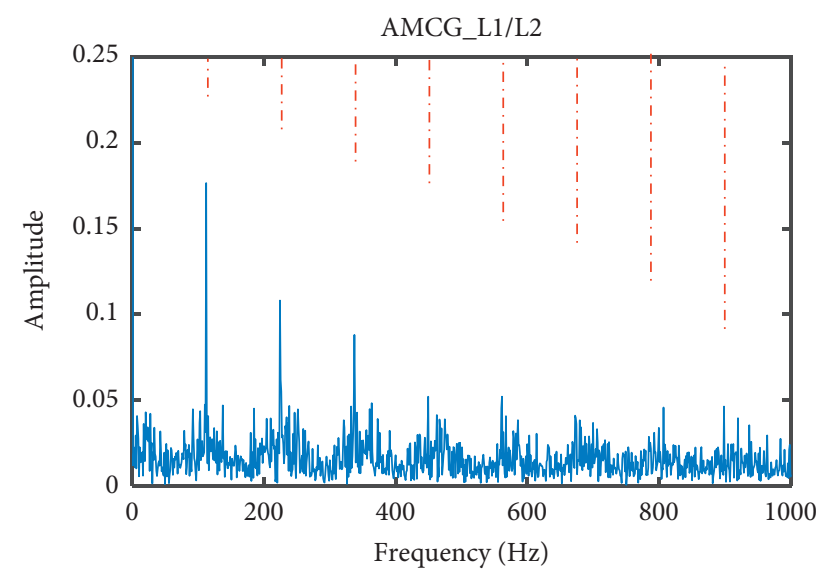

(b)

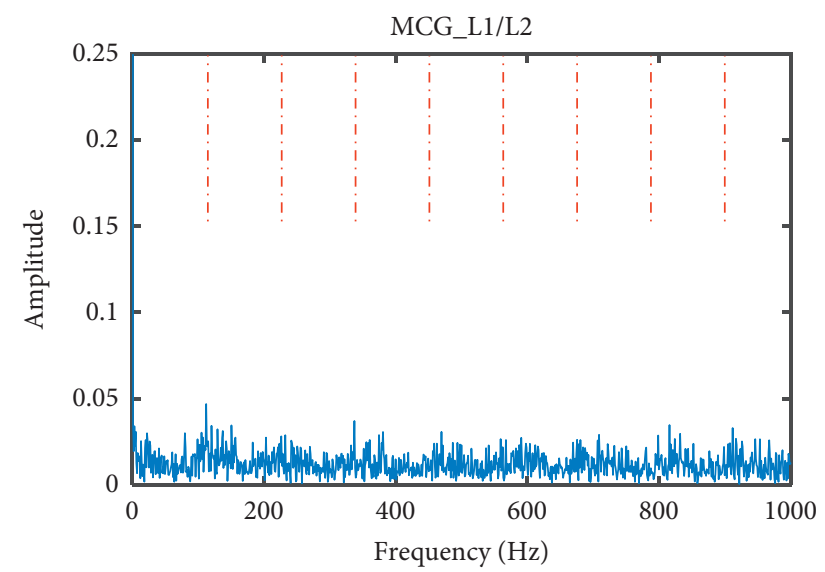

(d)

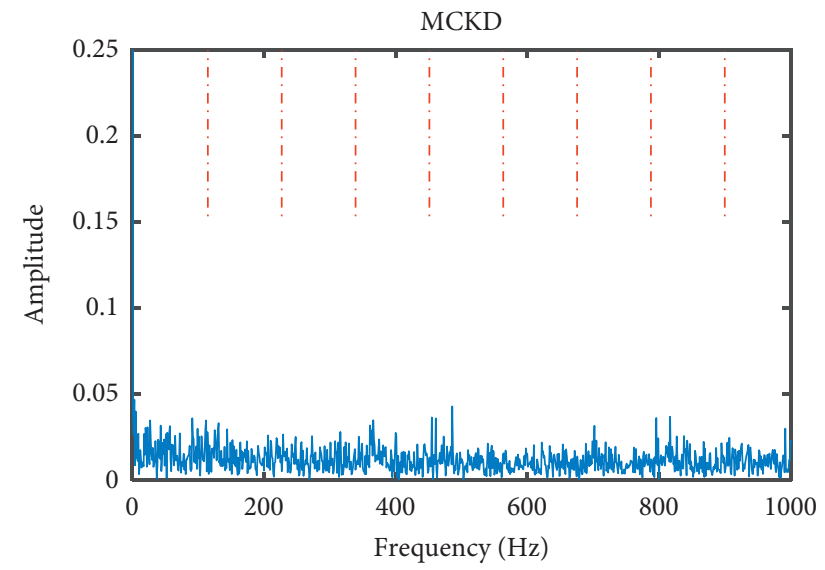

(f)

FIgure 6: Processing results of AMCG- $L p / L q-D$, MCG- $L p / L q-D$, and MCKD for single fault simulation vibration signal (with the input of $116 \mathrm{~Hz}$ ): (a, c, e) waveforms obtained by AMCG- $L p / L q-\mathrm{D}$, MCG- $L p / L q-\mathrm{D}$, and MCKD; $(\mathrm{b}, \mathrm{d}, \mathrm{f})$ corresponding envelope spectra.

technology, AMCG- $L p / L q-\mathrm{D}$ does take more time to complete the iteration than MCG- $L p / L q-\mathrm{D}$. However, there is no doubt that only the proposed method can successfully estimate the real fault period and achieve the best fault feature enhancement effect among four methods. This indicates that the proposed method can obtain better fault detection results without significantly increasing computational complexity.
In summary, IMCKD, MCKD, and MCG- $L p / L q-D$ cannot obtain satisfactory results when the SNR of simulation signal is low and the prior period is different from the real fault period. By comparing Figures 8(a) and 8(e), it can be seen that MCKD is sensitive to strong noise, resulting in its convergence to a single large peak. However, MCG- $L p /$ $L q-\mathrm{D}$ has a pretty good antinoise capability, which is the reason that MCG- $L p / L q-\mathrm{D}$ can still detect fault features 


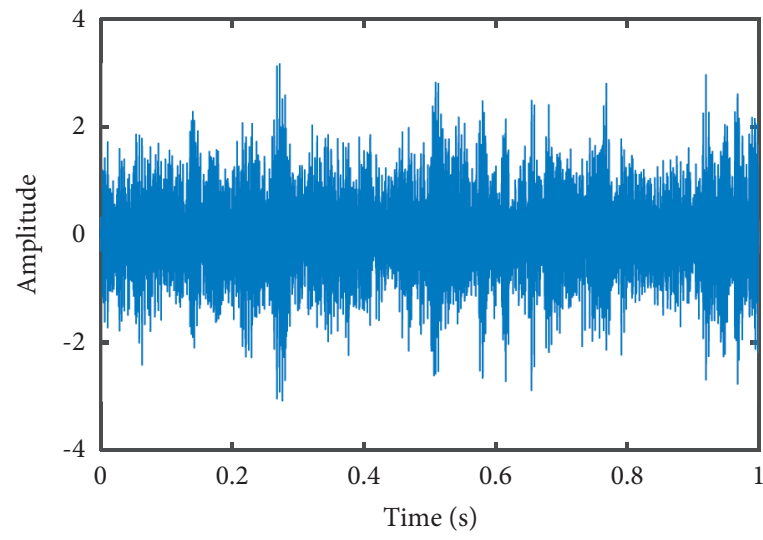

(a)

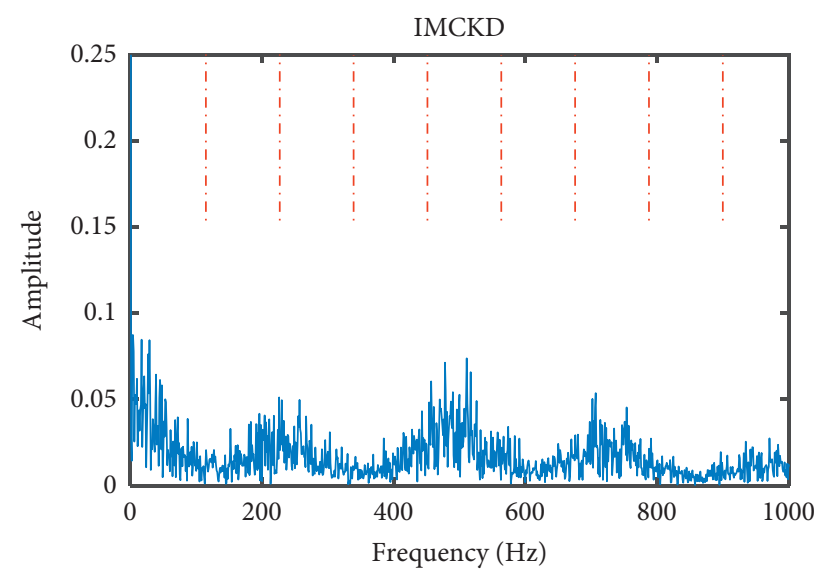

(b)

FIGURE 7: Processing result of IMCKD for single fault simulation vibration signal: (a) waveform; (b) envelope spectrum.

weakly even without precise prior period, as shown in Figure $8(\mathrm{~d})$. AMCG- $L p / L q$-D shows very strong fault features detection capability under the above two prior periods, and the period of its filtered signal converges to the real fault period. This indicates that the proposed APEC has stable period estimation capability under the condition of strong noise, which ensures that the deconvolution method converges to the correct result.

4.2. Concurrent Fault Simulation Signal Test. A concurrent simulation signal is used to verify the superiority of AMCG$L p / L q-\mathrm{D}$. The concurrent simulation vibration signal is made by adding a set of new single fault simulation impacts to the simulation signal in Figure 5(a). The parameters of the newly added simulation impacts are listed in Table 3. The timedomain waveforms of the simulation signal containing concurrent fault and its envelope spectrum are shown in Figure 10. In Figure 10(b), the red dotted line corresponds to $F_{d}=91 \mathrm{~Hz}$, while the yellow line corresponds to $F_{d}=112 \mathrm{~Hz}$. IMCKD, MCKD, and MCG- $L p / L q-\mathrm{D}$ are also used to deal with this signal. The results of these three methods are compared with that of the proposed AMCG-Lp/ $L q-\mathrm{D}$.

Firstly, the fault with feature frequency of $91 \mathrm{~Hz}$ is detected. The prior period is set as 105.3 and its corresponding frequency is $95 \mathrm{~Hz}$. The concurrent fault simulation vibration signal is processed by AMCG- $L p / L q-\mathrm{D}, M C G-L p / L q-\mathrm{D}$, and MCKD, and the results are shown in Figure 11. In Figure 11(b), it can be observed that AMCG- $L p / L q-\mathrm{D}$ accurately identifies the fault features corresponding to $91 \mathrm{~Hz}$ in the concurrent fault. In the meanwhile, according to Figure 12(a), after about nine iterations, APEC converges to $T_{e}=110 \approx 10000 / 91=T_{r}$ at the red dot mark. Although this fault is also identified by MCG- $L p / L q-\mathrm{D}$, its recovery performance of fault features is not ideal, as shown in Figure 11(d). MCKD converges to the single peaks in the signal and completely fails to recover the fault features submerged in the noise, as shown in Figure 11(f). This case demonstrates the importance of precise prior period to the performance of MCKD and MCG-Lp/Lq-D. However, the proposed method is very robust. Even if the input prior period parameters are imprecise, AMCG- $L p / L q-D$ can still obtain good results.

Secondly, the fault with feature frequency of $113 \mathrm{~Hz}$ is detected. The prior period is set as 87 and its corresponding frequency is $115 \mathrm{~Hz}$. The processing results of AMCG- $L p /$ $L q-\mathrm{D}, \mathrm{MCG}-L p / L q-\mathrm{D}$, and MCKD are shown in Figure 13. It can be found that only the proposed AMCG- $L p / L q-\mathrm{D}$ can clearly find the fault features corresponding to $113 \mathrm{~Hz}$ in the composite fault, whereas the other two methods cannot detect the fault features. The iteration process of APEC for AMCG- $L p / L q$-D is shown in Figure 12(b). It can also be clearly observed that APEC converges to $T_{e}=88 \approx 10000 / 113=T_{r}$ at the red dot. Additionally, according to the results shown in Figures 13(a), 13(c), 13(e), and 14(a), the time-domain waveforms of both MCKD and IMCKD have large peaks, whereas that of the proposed AMCG- $L p / L q$-D and MCG- $L p / L q-D$ does not have. This proves that AMCG- $L p / L q$-D and MCG- $L p / L q$-D have good robustness to strong noises. The comparison between Figures 13(b) and 13(d) shows that APEC can update the fault period in each iteration and promote AMCG- $L p / L q-D$ to converge to the correct result. However, MCG-Lp/Lq-D fails to detect any fault features, indicating that the performance of MCG- $L p / L q$-D is heavily dependent on the real period.

Since the concurrent fault has two different fault periods, IMCKD theoretically cannot detect two different periods at the same time. This is the main reason for the failure of IMCKD in this concurrent fault diagnosis task, as shown in Figure 14(b). The period variation of the filtering signal during its iteration is shown in Figure 12(c). Additionally, since APE performs global period detection, the estimated range of period fluctuation is too large, resulting in that IMCKD fails to converge, as shown in Figure 12(c). Therefore, IMCKD cannot detect any faults. By contrast, the results in Figures 9(a), 9(b), 12(a), and 12(b) show that the estimation period obtained by APEC rapidly converges to a stable value after a certain number of iterations. This makes 


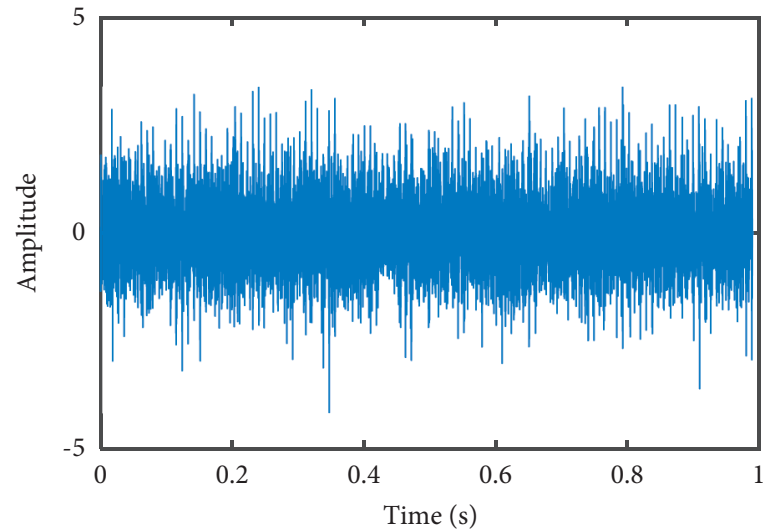

(a)

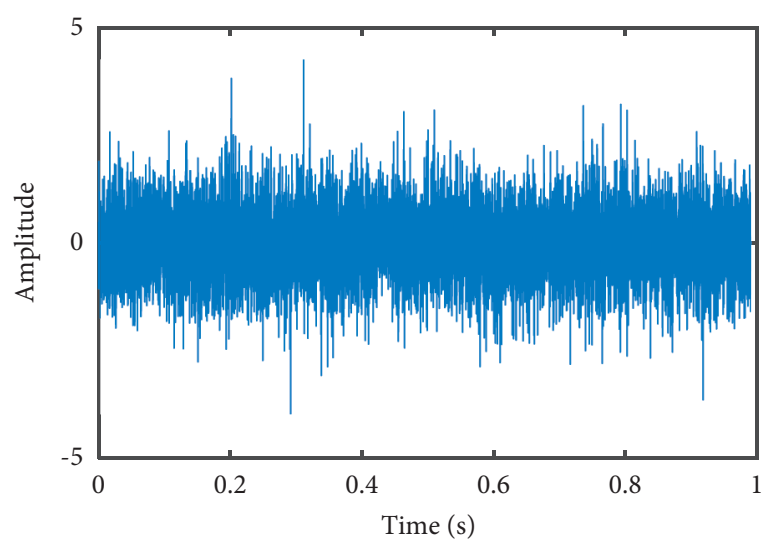

(c)

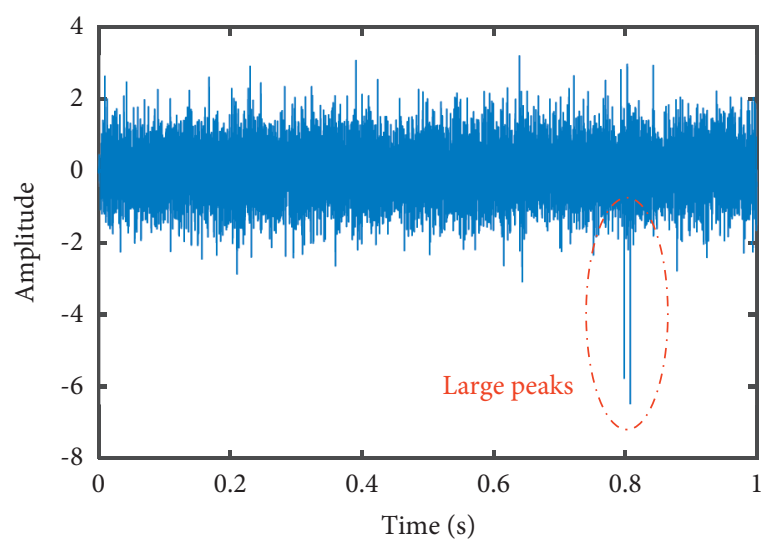

(e)

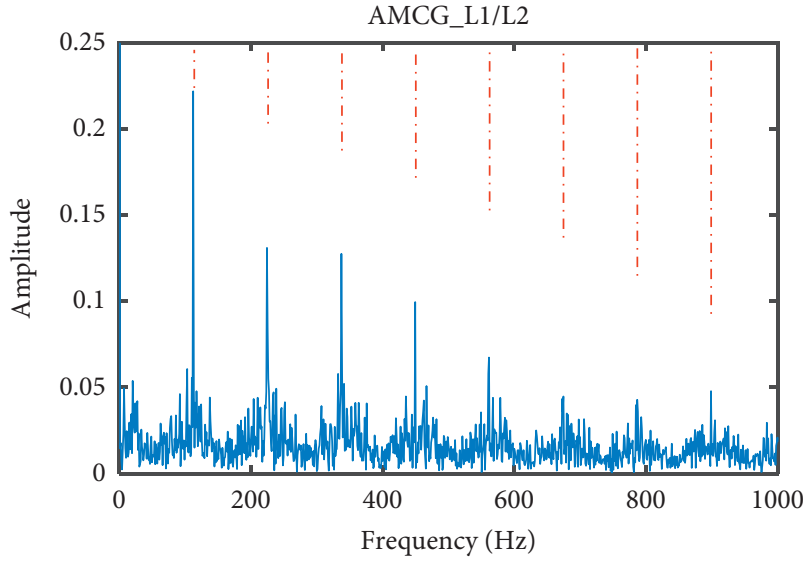

(b)

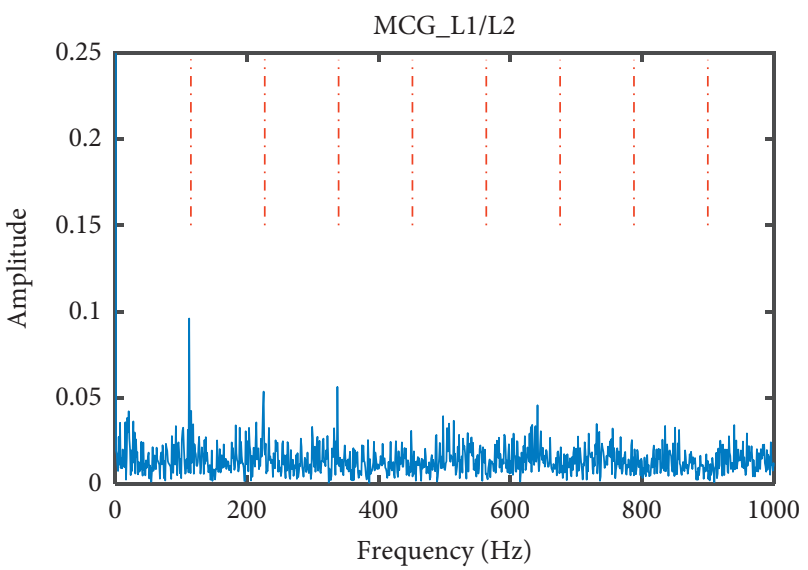

(d)

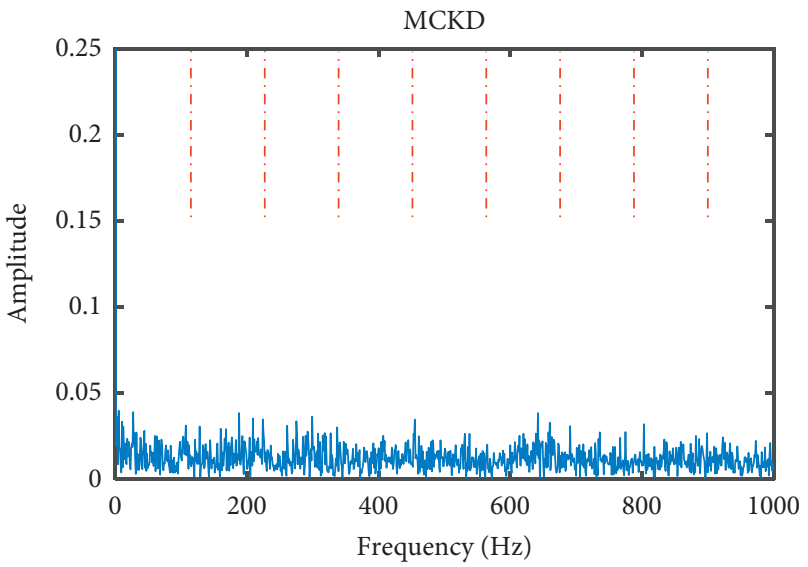

(f)

FIGURE 8: Processing results of AMCG- $L p / L q-D$, MCG- $L p / L q-D$, and MCKD for single fault simulation vibration signal (with the input of $108 \mathrm{~Hz}$ ): (a, c, e) waveforms obtained by AMCG- $L p / L q-D$, MCG- $L p / L q-D$, and MCKD; $(\mathrm{b}, \mathrm{d}, \mathrm{f})$ corresponding envelope spectra.

AMCG- $L p / L q-D$ have a strong capability to detect the real fault period when dealing with the signal containing single or concurrent faults.

The simulation case study shows that the proposed AMCG- $L p / L q$-D exhibits very strong robustness under the condition of strong noise and when there is a difference between the given prior period and the real period. However, none of the other three methods can achieve good results. IMCKD completely modifies MCKD to a nonperiod dependent method, which makes IMCKD unable to realize concurrent fault detection. Additionally, due to the influence of strong noise, its period estimation method, APE, cannot find the fault period accurately, which eventually leads to IMCKD unable to converge. 


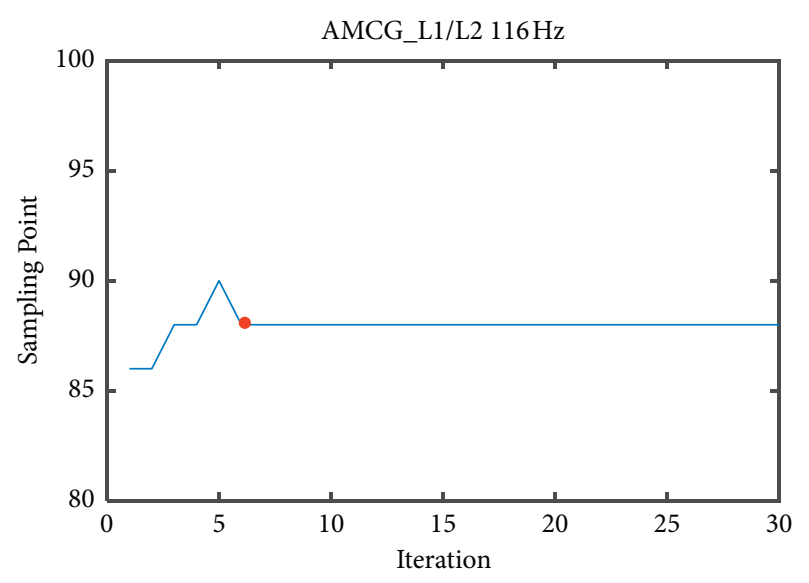

(a)

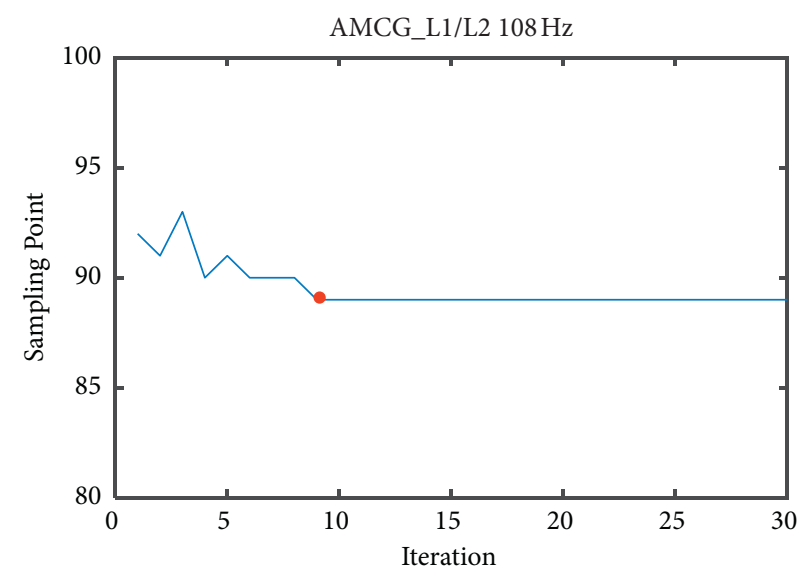

(b)

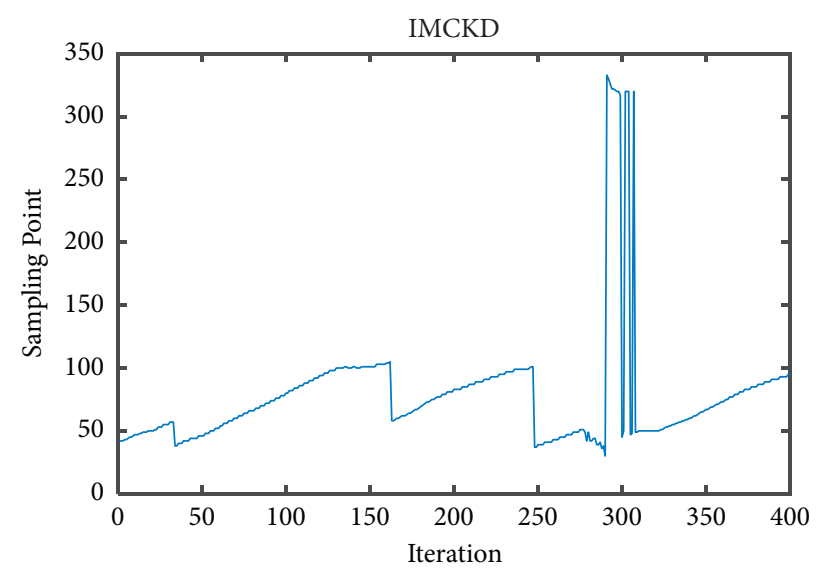

(c)

FIGURE 9: Period estimation of AMCG- $L p / L q-D$ and IMCKD for single fault simulation vibration signal: (a) AMCG- $L p / L q$-D with the input of $116 \mathrm{~Hz}$; (b) AMCG- $L p / L q-\mathrm{D}$ with the input of $108 \mathrm{~Hz}$; (c) IMCKD.

TABLE 2: Computational efficiency.

\begin{tabular}{lccc}
\hline AMCG-L1/L2-D (s) & MCG-L1/L2-D (s) & MCKD (s) & IMCKD (s) \\
\hline 23.83 & 20.68 & 19.97 & 24.52
\end{tabular}

TABLe 3: Parameters of newly added single fault simulation vibration signal.

\begin{tabular}{ccccc}
\hline$A$ & $\beta$ & $f_{R S}(\mathrm{~Hz})$ & $F_{d}(\mathrm{~Hz})$ & $f_{r}(\mathrm{~Hz})$ \\
\hline 1 & 800 & 1600 & 91 & 10.29 \\
\hline
\end{tabular}

\section{Experimental Case Studies}

In this section, a group of single fault experimental data and a group of concurrent fault experimental data are used to test the proposed method to verify its application value in engineering practice. Both sets of experimental data were obtained through the test rig shown in Figure 15(a). The main components of the test rig are motor, testing wheelset, driving wheels, and axle box. The bearings tested are axle box bearings of high-speed railway vehicles, which belong to double-row tapered roller bearings.
Table 4 demonstrates the geometric dimensions of the bearings used, where $d_{P}(\mathrm{~mm})$ is the pitch diameter, $d_{R}(\mathrm{~mm})$ is the roller-ball diameter, $Z$ is the number of balls, and $\theta(\mathrm{rad})$ is the contact angle of balls. The test wheelset is driven by the driving wheel at the bottom and supported by the axle box bearing. Figure 15(b) shows the installation position on the axle box of the accelerometer used to collect the vibration mixture signals. An ZW9609A-18SN7068 accelerometer for collecting vibration signals is mounted on the axle box, as shown in Figure 15(b). Figures 15(c) and 15(d) separately demonstrate the obvious outer-race faults and rolling element faults of the two tested bearings. It is worth noting that the bearing with rolling element fault also has obvious outer-race fault. Both sets of experimental data were obtained at speeds close to $100 \mathrm{~km} / \mathrm{h}$ (rotation frequency is $10.29 \mathrm{~Hz}$ ). The feature frequencies corresponding to the outer-race fault and the rolling element fault are $83.33 \mathrm{~Hz}$ and $67.86 \mathrm{~Hz}$, respectively.

It must be noted that the test rig experiment has the conditions to precisely collect the relevant parameters used in precise calculation of the real fault feature frequency. However, in engineering practices, the acquisition of some parameters is restricted by the testing environment, resulting 


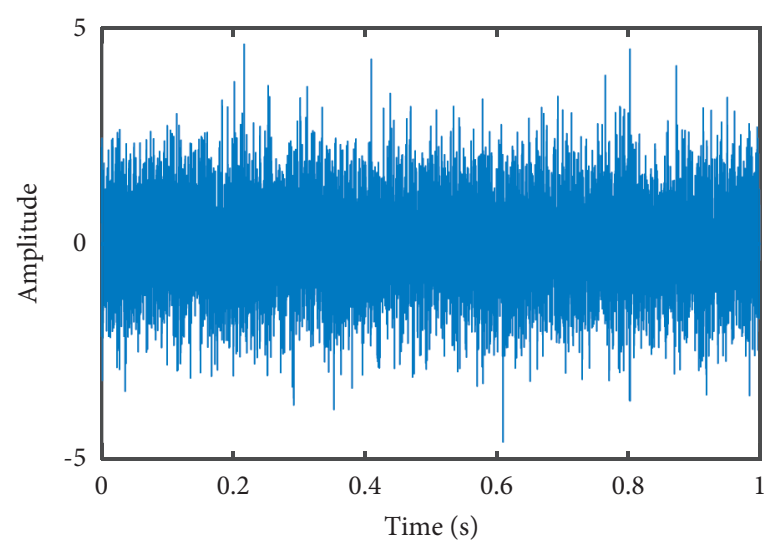

(a)

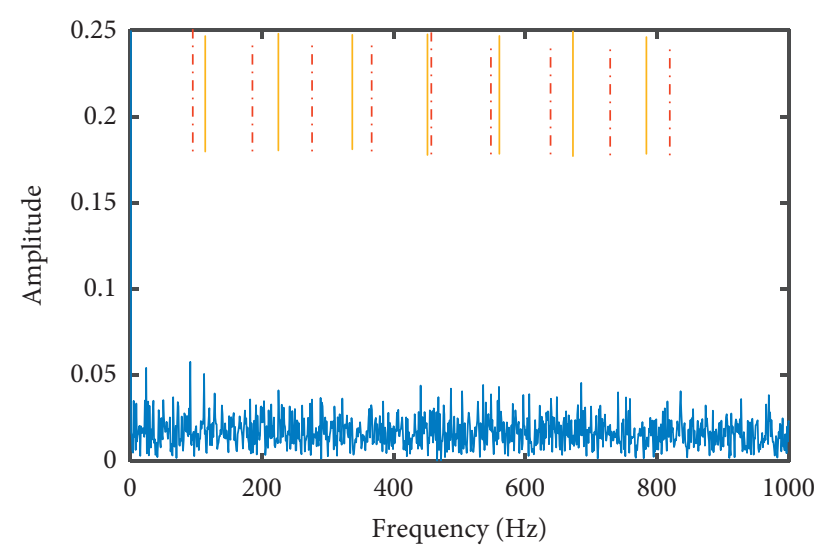

(b)

FIGURE 10: Concurrent fault simulation vibration signal: (a) waveform; (b) envelope spectrum.

in that they cannot be precisely obtained. In order to verify the superiority of the proposed method in engineering practices, in the case studies of this section, the fault feature frequency will be calculated by using some values deviating from the real speed instead of using the real speed. This is used to simulate the possible speed measurement error in practical engineering.

5.1. Single-Fault Experimental Data Test. The single outerrace fault experimental vibration signal and its envelope spectrum of the bearing outer-race fault are shown in Figure 16. The red dotted line in Figure 16(b) marks the feature frequency of the bearing outer-race fault. When the operation speed is $106 \mathrm{~km} / \mathrm{h}$ (the corresponding shaft rotation frequency is $10.87 \mathrm{~Hz}$ ), the input prior period is set as 113.7, and the corresponding frequency of this period is $88 \mathrm{~Hz}$. Figures 17 and 18 show the processing results of AMCG- $L p / L q-D$, MCG- $L p / L q-D, M C K D$, and IMCKD. It can be found that when the prior fault feature frequency is set to $88 \mathrm{~Hz}$ higher than the real fault feature frequency, the proposed AMCG- $L p / L q-\mathrm{D}$ precisely detects the real fault features and greatly improves its amplitude. This can be observed by making comparison among the envelope spectra before and after processing in Figures 16(b), 17(b), 17(d), 17(f), and 18(b). All other methods failed. The APEC variation of the proposed method in the iteration process is shown in Figure 19(a). It can be observed that it converges to $T_{e}=119 \approx 10000 / 83.33=T_{r}$. Although MCG- $L p / L q-\mathrm{D}$ also detects the fault features, its recovery performance is far from that of the proposed AMCG-Lp/Lq-D. By contrast, MCKD and IMCKD are completely unable to detect the fault features.

The case study shows that the performance of fault feature detection for MCG-Lp/Lq-D and MCKD will be seriously affected if the precise prior period of bearing fault cannot be provided under strong noise interference. Due to the defects of APE, IMCKD cannot accurately detect the real fault period, resulting in that the algorithm fails to converge, as shown in Figure 19(b). By contrast, the APEC in the proposed AMCG- $L p / L q-D$ can approach the real fault period continually in the iteration and finally makes AMCG$L p / L q$-D converge to the real period, so as to detect and enhance the fault features. This conclusion is the same as that obtained in the simulation analysis of bearing single fault in Section 4.1.

In order to test whether the computational complexity of the proposed method in engineering practice is consistent with the conclusion, related to computational complexity, obtained in the simulated case study, the computational efficiency of the four methods is given in Table 5. AMCG- $L p / L q-D$, MCG- $L p / L q-D, M C K D$, and IMCKD consume $20.03 \mathrm{~s}, 14.47 \mathrm{~s}, 13.12 \mathrm{~s}$, and $24.52 \mathrm{~s}$, respectively, for iterative calculation. It can be seen that the calculation time of AMCG- $L p / L q-D$ and IMCKD is indeed longer than that of MCG-Lp/Lq-D and MCKD due to the addition of adaptive period estimation technology. However, only AMCG- $L p / L q-\mathrm{D}$ accurately estimates the real fault period and finally detected the outer-race fault features through 22 iterations when the input period deviates significantly from the real fault period. By contrast, IMCKD takes longer calculation time, and its period detection effect is not ideal because of serious noise interference. This further indicates that APEC used in the proposed method has better period tracking ability in engineering practice than APE used in IMCKD, especially in the case of strong background noise.

5.2. Concurrent Fault Experimental Data Test. A set of concurrent fault experimental vibration signals is used to verify the concurrent fault diagnosis capability of AMCG$L p / L q$-D in engineering practice. Figures 20 (a) and 20 (b) separately show the time-domain waveform and the envelope spectrum of the collected concurrent fault experimental vibration signal. In Figure 20(b), the red dotted line corresponds to the bearing outer-race fault of $83.33 \mathrm{~Hz}$, and the yellow line corresponds to the bearing rolling element fault of $67.86 \mathrm{~Hz}$. In the envelope spectrum, only the fundamental frequency of the feature frequency of the outer-race fault can be observed, but neither its harmonic nor the rolling element fault can be detected. 


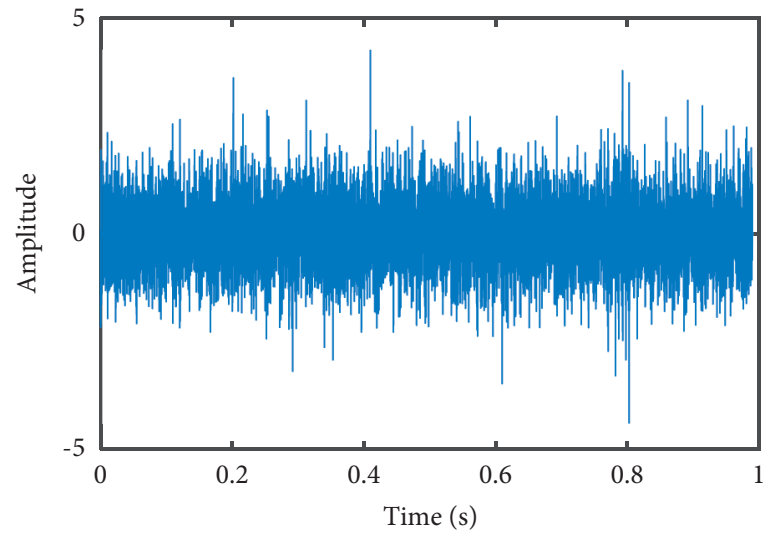

(a)

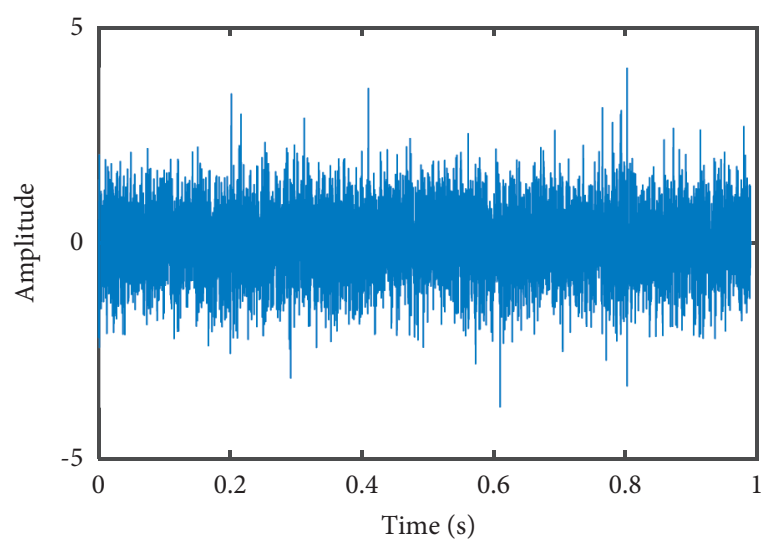

(c)

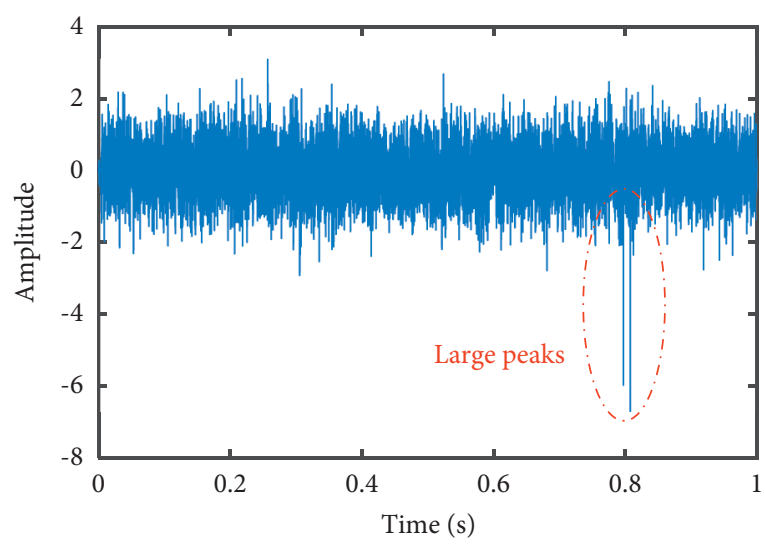

(e)

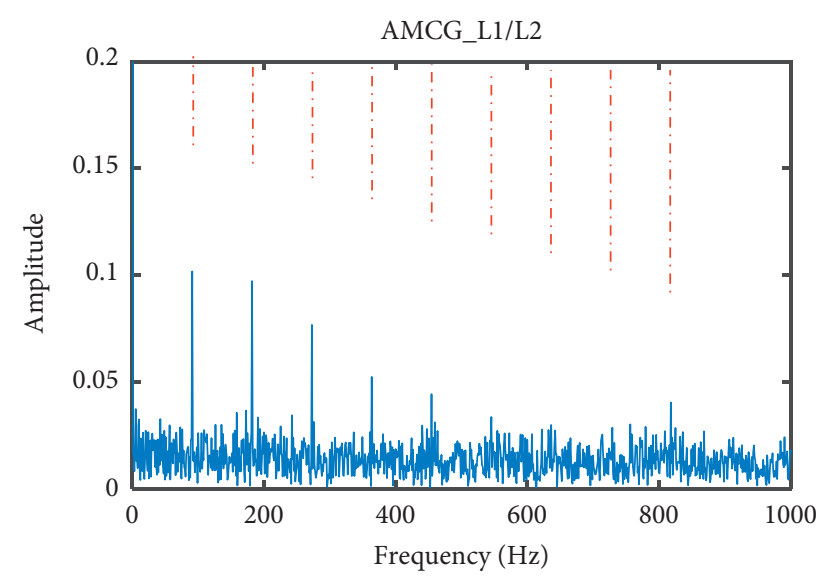

(b)

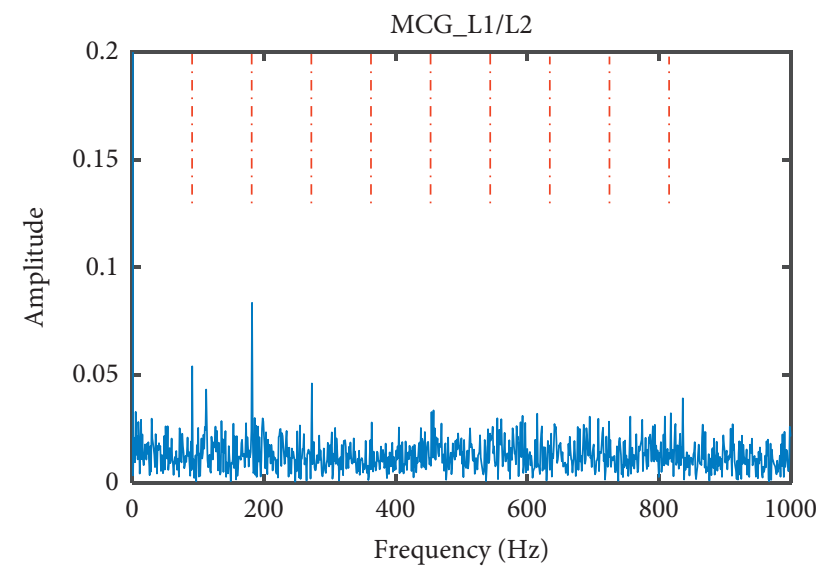

(d)

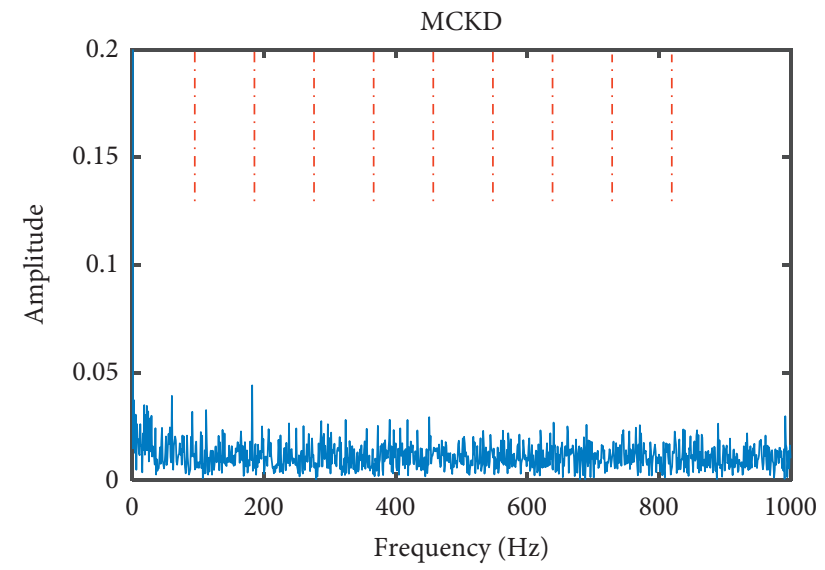

(f)

FIGURe 11: Processing results of AMCG- $L p / L q-D$, MCG- $L p / L q-D$, and MCKD for concurrent fault simulation vibration signal (with the input of $95 \mathrm{~Hz}$ ): (a, c, e) waveforms obtained by AMCG- $L p / L q-\mathrm{D}, \mathrm{MCG}-L p / L q-\mathrm{D}$, and MCKD; (b, d, f) corresponding envelope spectra.

Firstly, the rolling element fault is detected. When the operation speed is $105 \mathrm{~km} / \mathrm{h}$ (the corresponding shaft rotation frequency is $10.84 \mathrm{~Hz}$ ), the prior period of AMCG- $L p /$ $L q-\mathrm{D}, \mathrm{MCG}-L p / L q-\mathrm{D}$, and MCKD is set as 140.85 and the corresponding frequency is $71 \mathrm{~Hz}$. This frequency deviates from the real fault feature frequency of $67.86 \mathrm{~Hz}$, about $3 \mathrm{~Hz}$. Figures 21 and 22 show the processing results of AMCG- $L p /$ $L q-\mathrm{D}, \mathrm{MCG}-L p / L q-\mathrm{D}, \mathrm{MCKD}$, and IMCKD. When the prior fault feature frequency is set to $71 \mathrm{~Hz}$, it can be observed from Figure 21 that only the proposed AMCG- $L p / L q-\mathrm{D}$ can accurately detect the $67.86 \mathrm{~Hz}$ bearing rolling element fault, whereas MCG- $L p / L q-\mathrm{D}$ and MCKD cannot identify any features related to the bearing rolling element fault. According to the iteration process in Figure 23(a), APEC finally converges to $T_{e}=146 \approx 10000 / 67.86=T_{r}$ at the red point. 


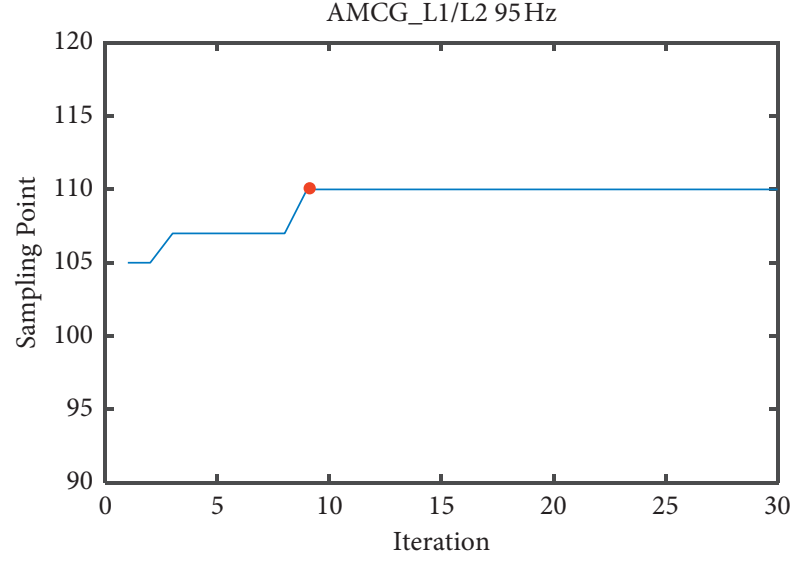

(a)

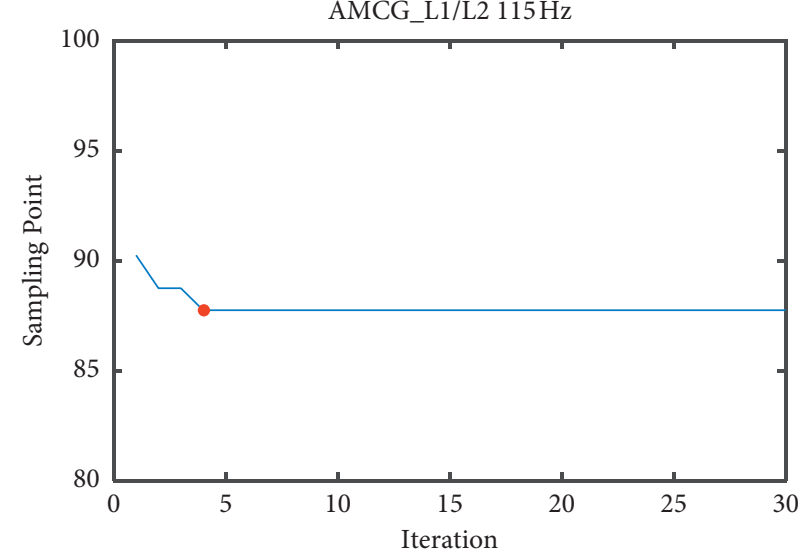

(b)

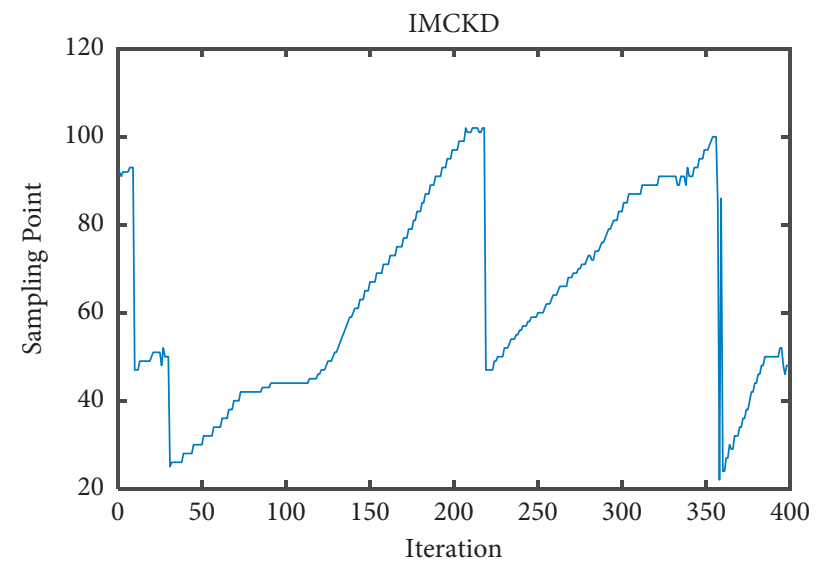

(c)

FIGURE 12: Period estimation of AMCG- $L p / L q$-D and IMCKD for concurrent fault simulation vibration signal: (a) AMCG- $L p / L q$-D with the input of $95 \mathrm{~Hz}$ (to detect the fault of $91 \mathrm{~Hz}$ ); (b) AMCG-Lp/Lq-D with the input of $115 \mathrm{~Hz}$ (to detect the fault of $113 \mathrm{~Hz}$ ); (c) IMCKD.

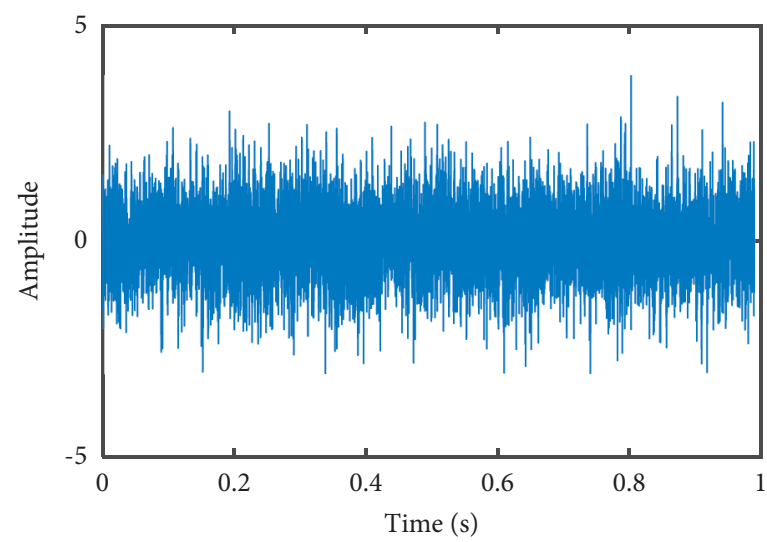

(a)

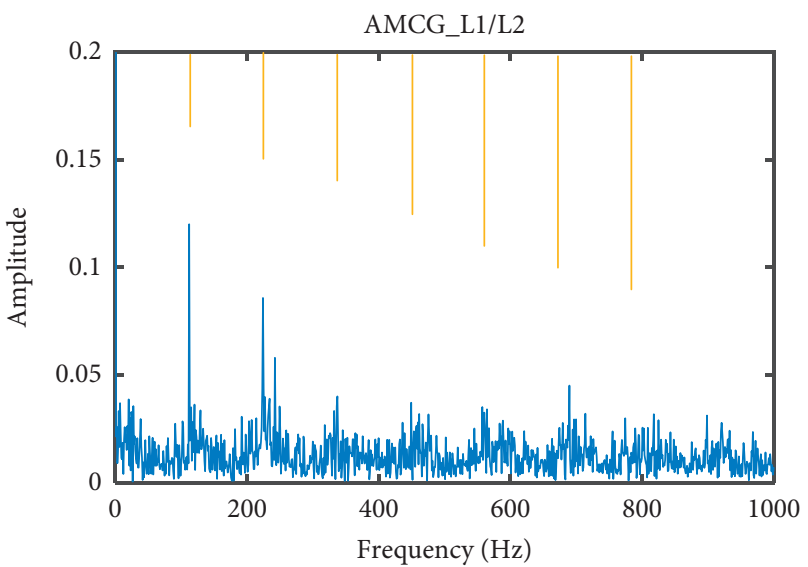

(b)

Figure 13: Continued. 


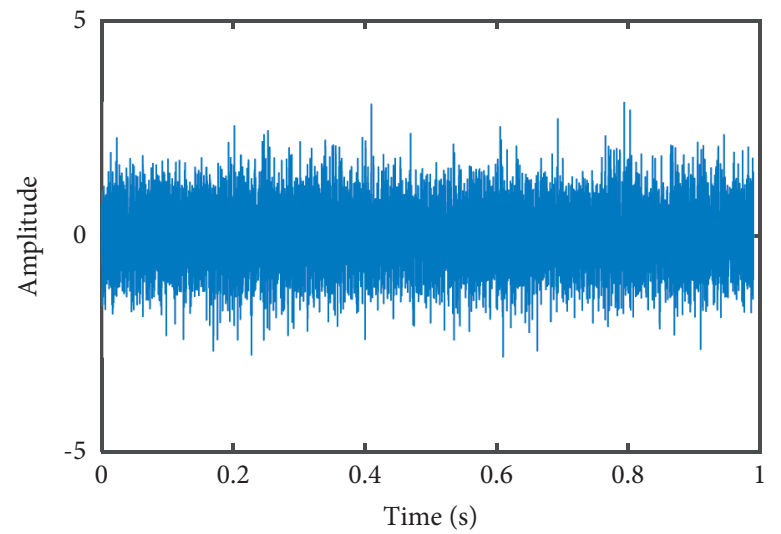

(c)

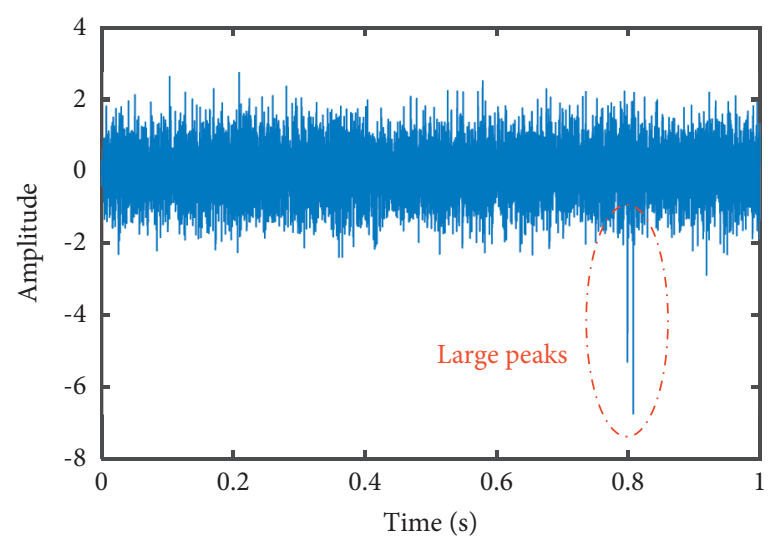

(e)

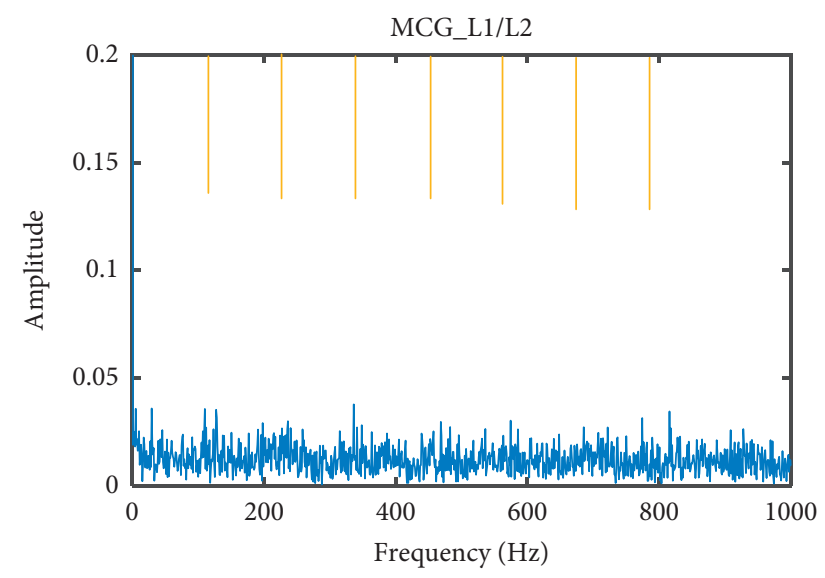

(d)

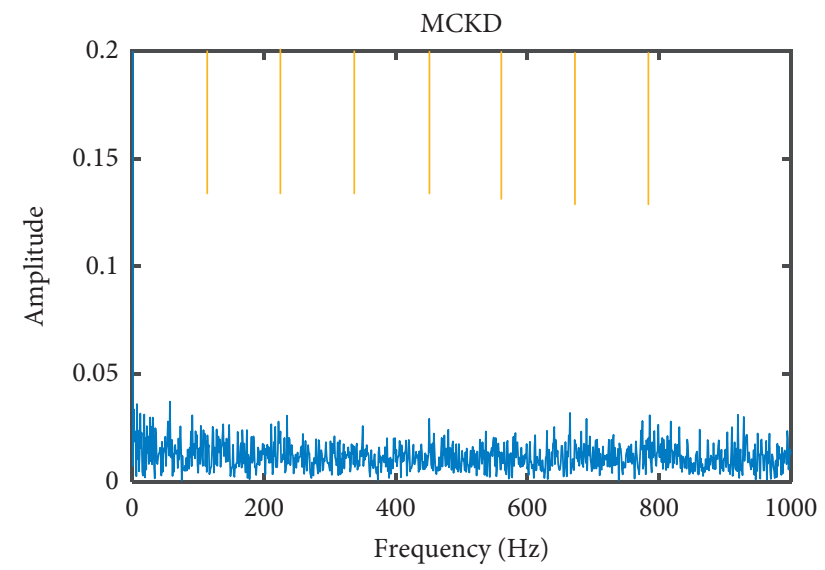

(f)

Figure 13: Processing results of AMCG- $L p / L q-D$, MCG- $L p / L q-D$, and MCKD for concurrent fault simulation vibration signal (with the input of $115 \mathrm{~Hz}$ ): (a, c, e) waveforms obtained by AMCG- $L p / L q-\mathrm{D}$, MCG-Lp/Lq-D, and MCKD; (b, d, f) corresponding envelope spectra.

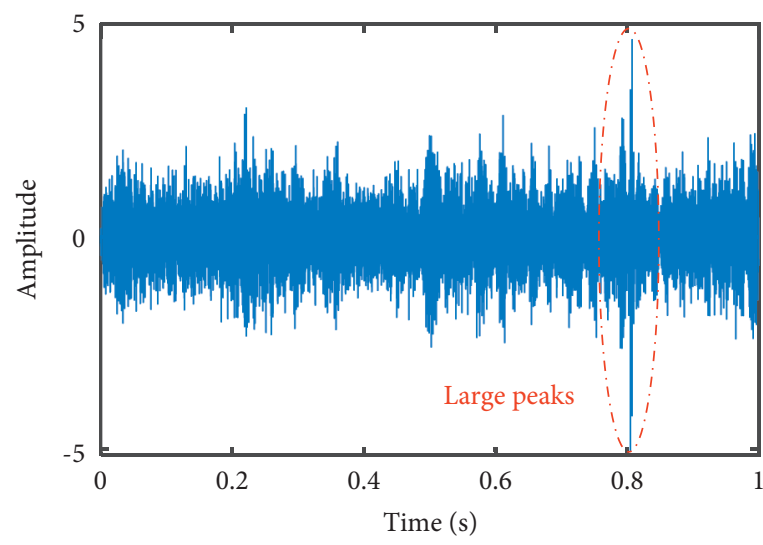

(a)

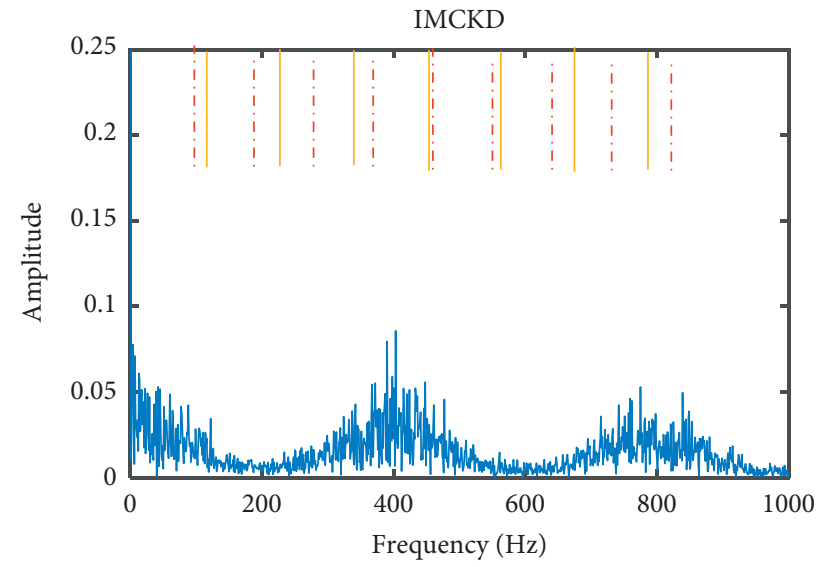

(b)

FIgURE 14: Processing result of IMCKD for concurrent fault simulation vibration signal: (a) waveform; (b) envelope spectrum.

Secondly, the outer-race fault is detected. The prior period of AMCG- $L p / L q-\mathrm{D}$, MCG- $L p / L q-\mathrm{D}$, and MCKD is set as 114.94 and the corresponding frequency is $87 \mathrm{~Hz}$. The processing results of the three methods are shown in
Figure 24. By comparing the processing results in Figures 24(b), 24(d), and 24(f), it can be found that MCG$L p / L q$-D and MCKD cannot detect the fault features of the bearing outer-race. Only the proposed AMCG- $L p / L q-\mathrm{D}$ can 


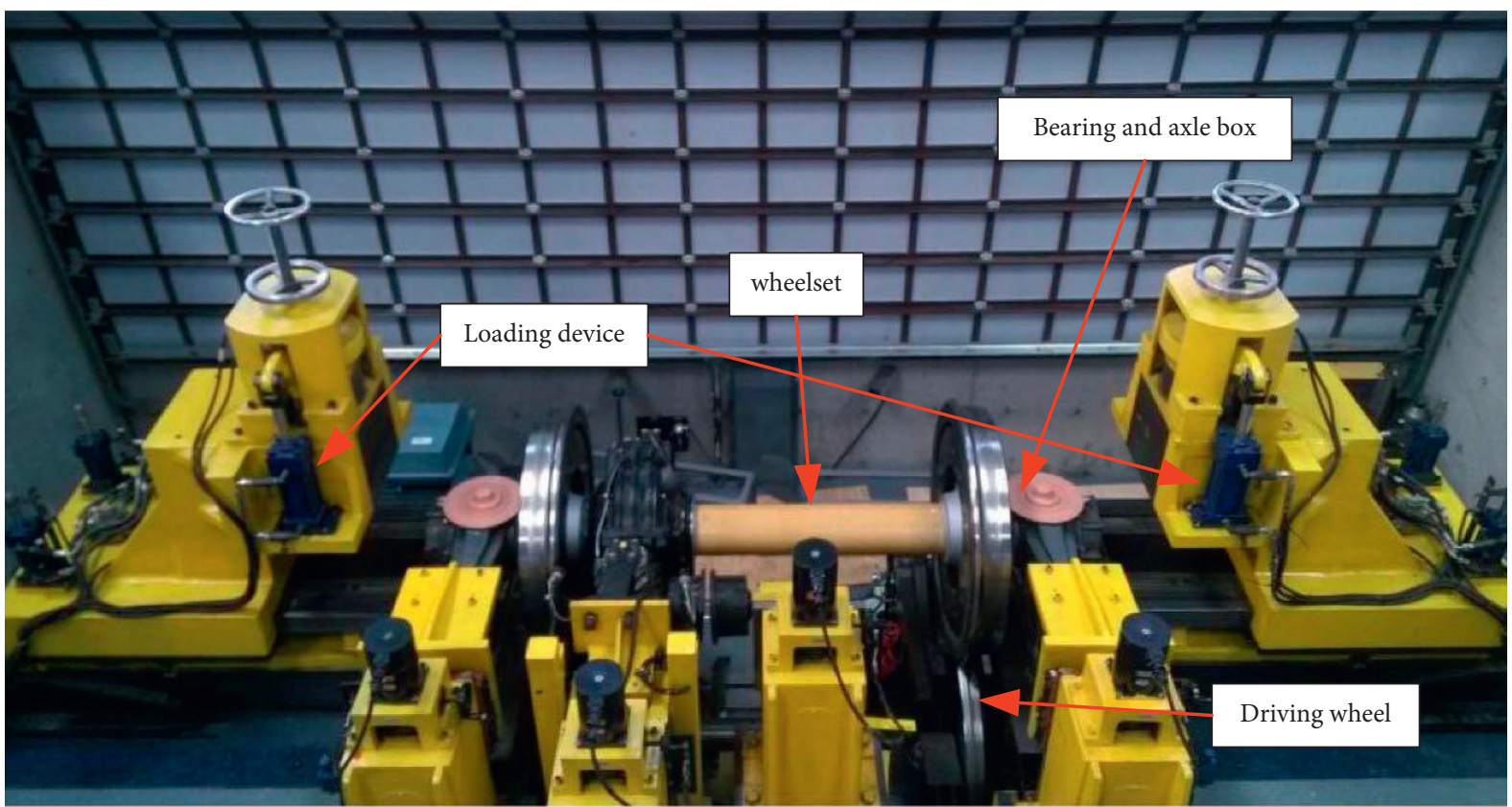

(a)

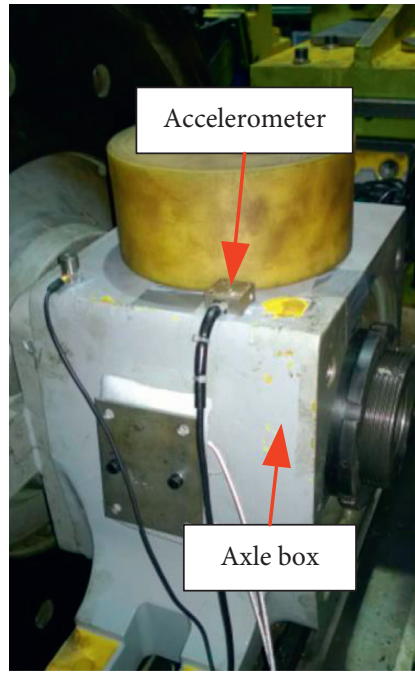

(b)

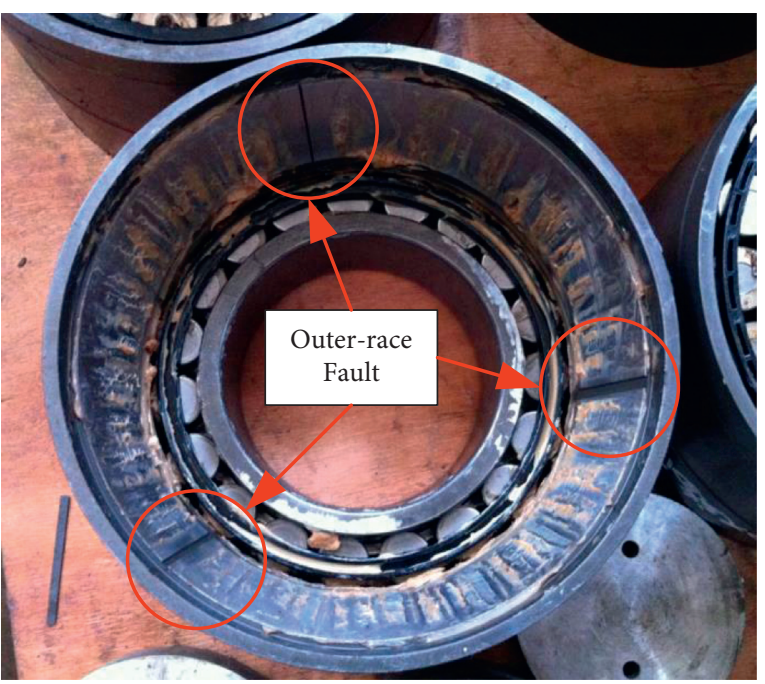

(c)

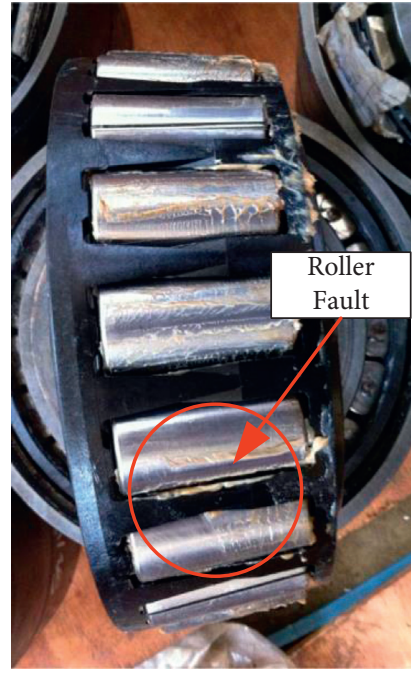

(d)

FIGURE 15: Photographs of bearing test rig: (a) testing rig; (b) installation of accelerometer; (c) outer-race fault; (d) rolling element fault.

TABLE 4: Bearing fault experimental data parameters.

\begin{tabular}{lccr}
\hline$d_{R}(\mathrm{~mm})$ & $d_{P}(\mathrm{~mm})$ & $Z$ & $\theta(\mathrm{rad})$ \\
\hline 26.9 & 180 & 19 & 0.1571 \\
\hline
\end{tabular}

extract the bearing outer-race fault features from the concurrent fault and enhance its amplitude. In addition, as shown in Figure 23(b), the APEC of the proposed method stabilizes to $T_{e}=119 \approx 10000 / 83.33=T_{r}$ at the red point after about 20 iterations. Figures 22(a) and 22(b) demonstrate the processing result of IMCKD. Since IMCKD does not have the ability to detect multiple faults, the failure of IMCKD in this experimental case is reasonable. It can be seen from Figure 24(c) that the fluctuation range of the period obtained by APE is too large to converge, denoting that detecting period in the global range might lead to nonconvergence of the result. This indicates that even if the prior period is imprecise, the theoretical calculation period can still guide AMCG- $L p / L q-\mathrm{D}$ to converge to the real result, and the concurrent fault detection capability of the proposed method is retained. 


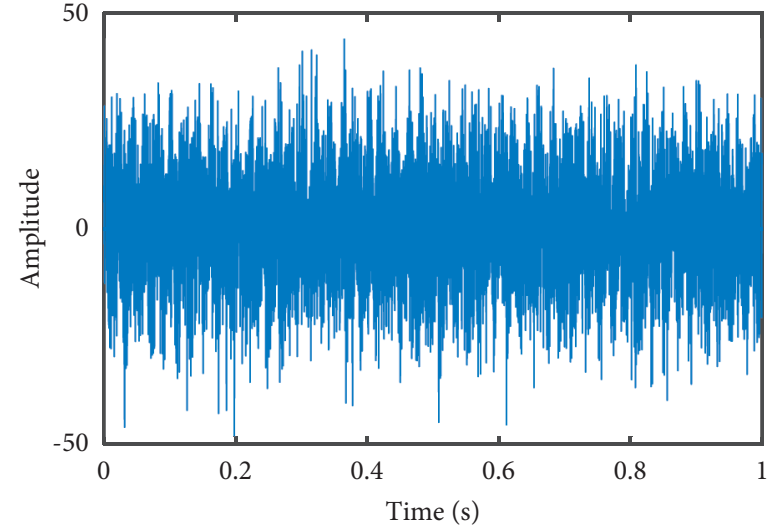

(a)

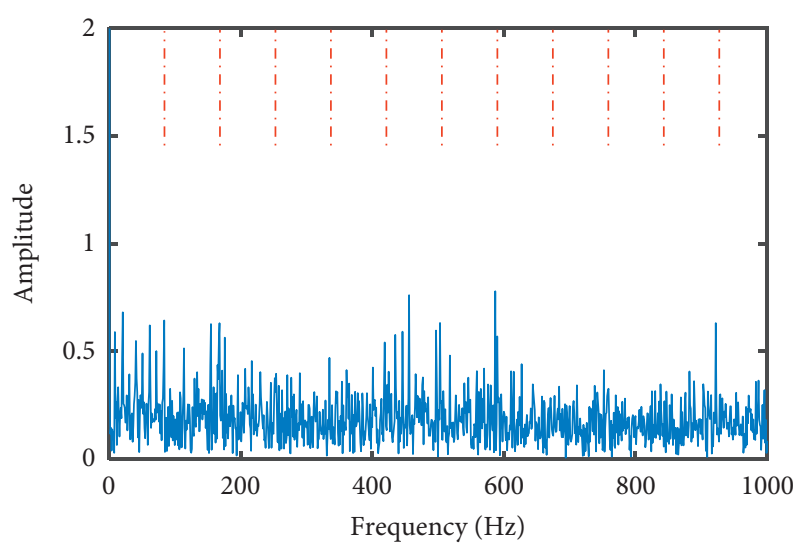

(b)

FIGURE 16: Single outer-race fault experimental vibration signal: (a) waveform; (b) envelope spectrum.

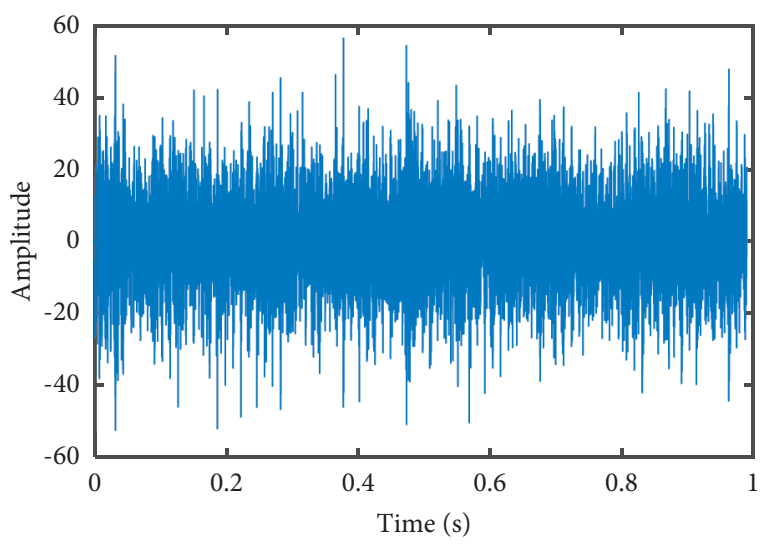

(a)

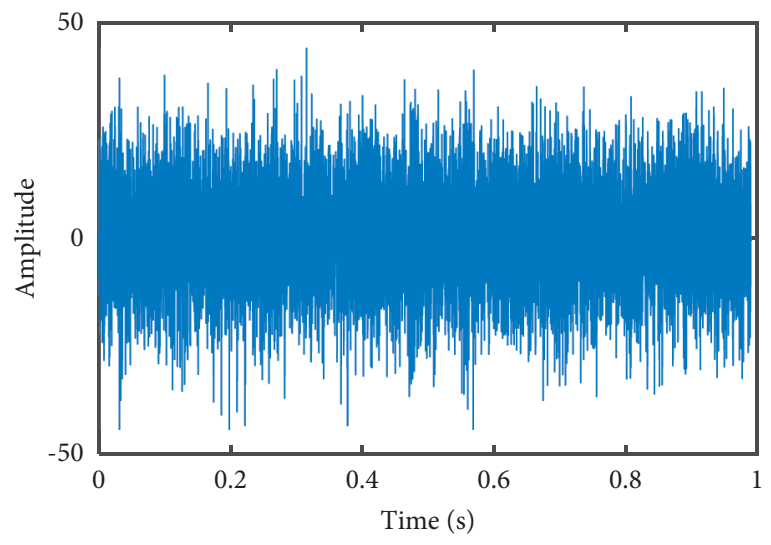

(c)

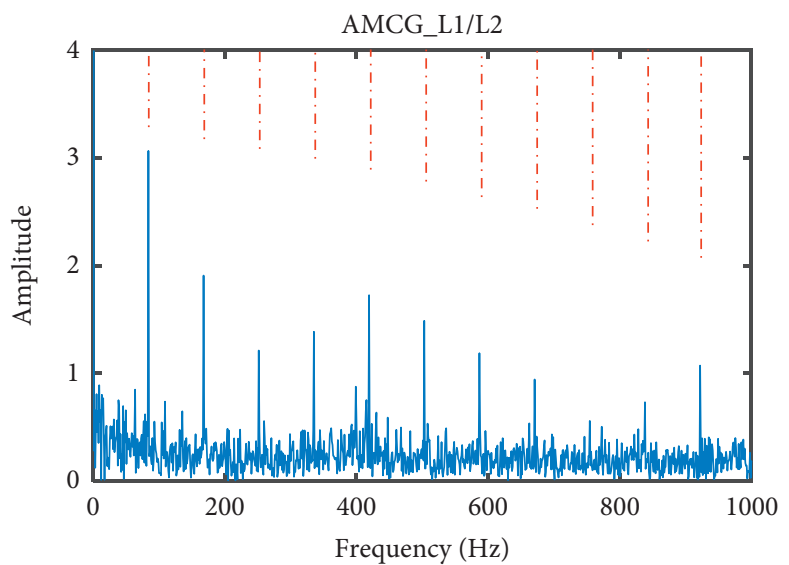

(b)

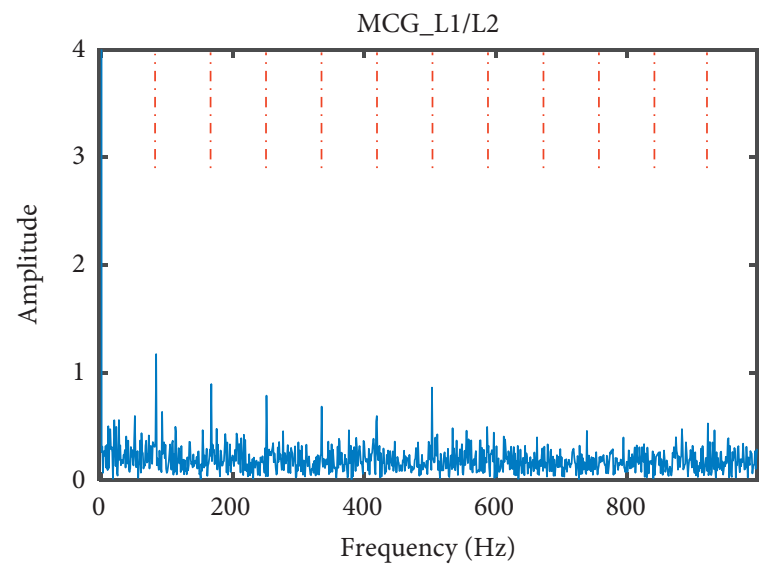

(d)

FIGURE 17: Continued. 


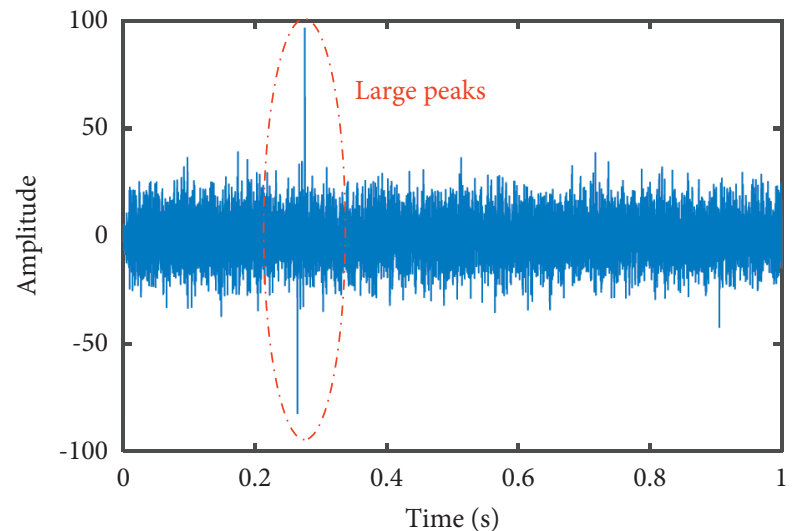

(e)

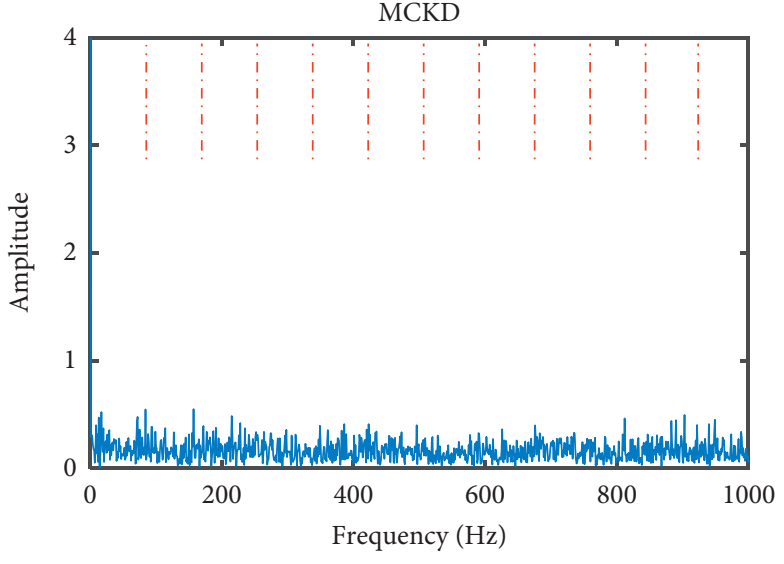

(f)

FIgURE 17: Processing results of AMCG- $L p / L q-\mathrm{D}$, MCG- $L p / L q-\mathrm{D}$, and MCKD for single outer-race fault experimental vibration signal (with the input of $88 \mathrm{~Hz}$ ): (a, c, e) waveforms obtained by AMCG-Lp/Lq-D, MCG- $L p / L q-\mathrm{D}$, and MCKD; (b, d, f) corresponding envelope spectra.

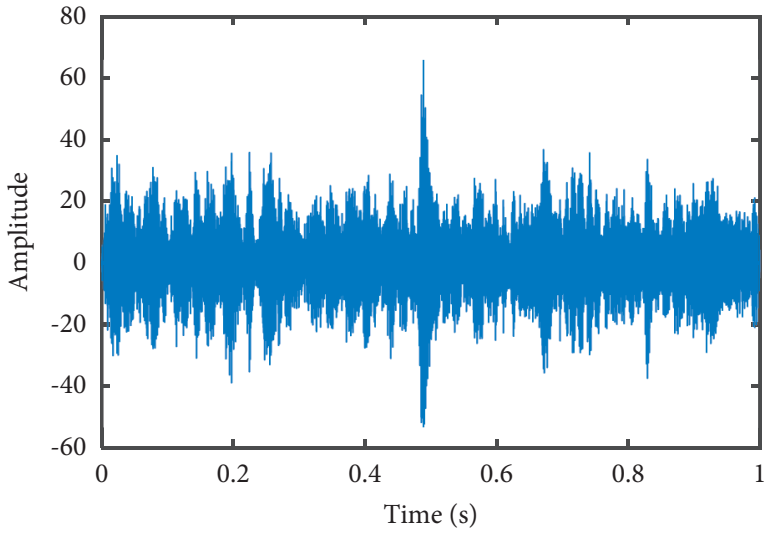

(a)

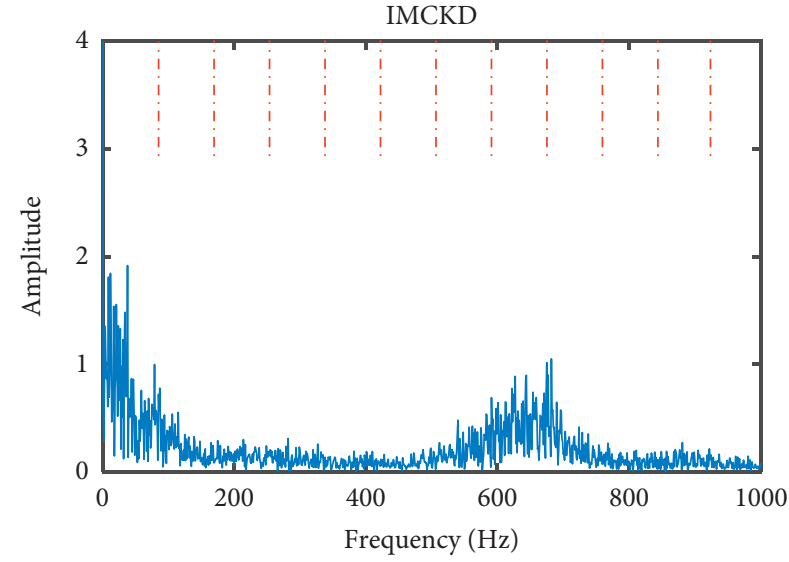

(b)

FIGURE 18: Processing results of IMKCD for single outer-race fault experimental vibration signal: (a) waveform; (b) envelope spectrum.

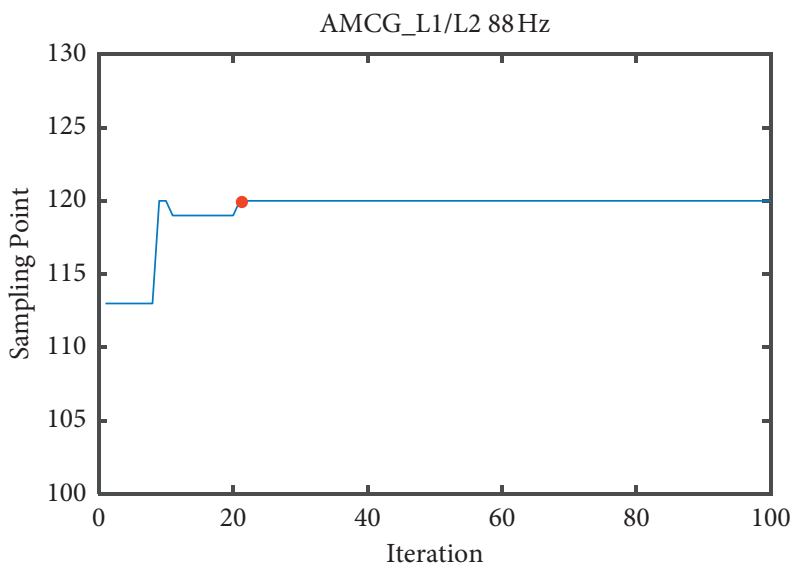

(a)

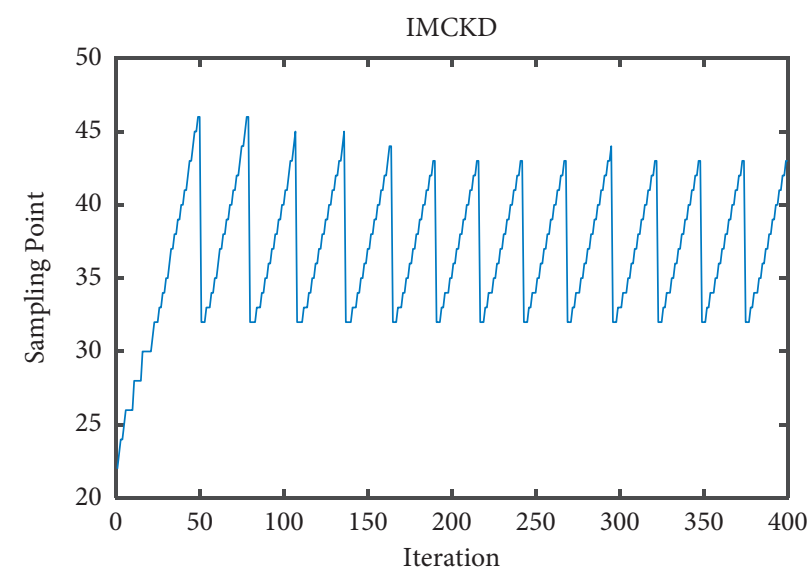

(b)

FIGURE 19: Period estimation of AMCG- $L p / L q-D$ and IMCKD for single outer-race fault experimental vibration signal: (a) AMCG- $L p / L q-D$ with the input of $88 \mathrm{~Hz}$; (b) IMCKD. 
TABLE 5: Computational efficiency.

\begin{tabular}{lccc}
\hline AMCG-L1/L2-D (s) & MCG-L1/L2-D (s) & MCKD (s) & IMCKD (s) \\
\hline 20.03 & 14.47 & 13.12 & 24.52 \\
\hline
\end{tabular}

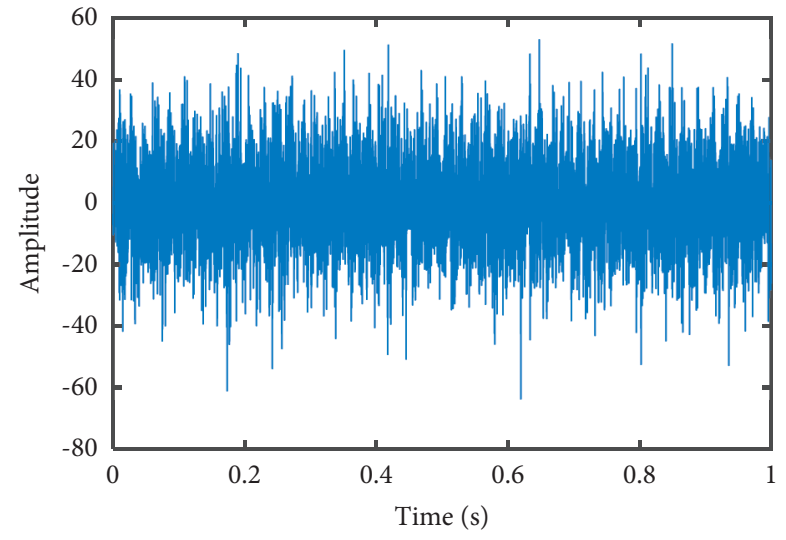

(a)

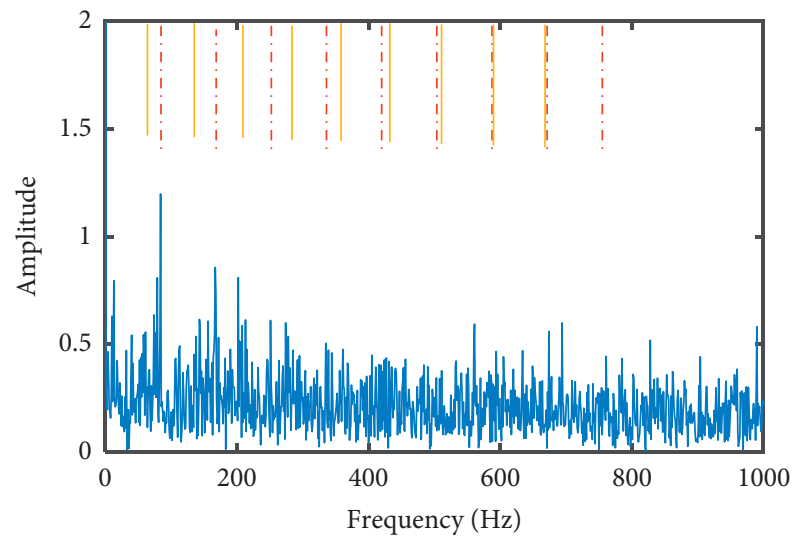

(b)

FIGURE 20: Experimental vibration mixture signal containing the concurrent fault of outer-race and rolling element: (a) waveform; (b) envelope spectrum.

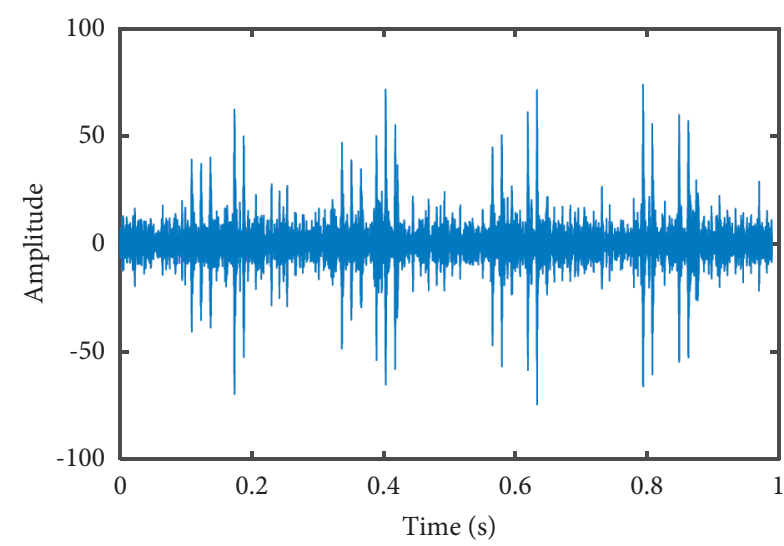

(a)

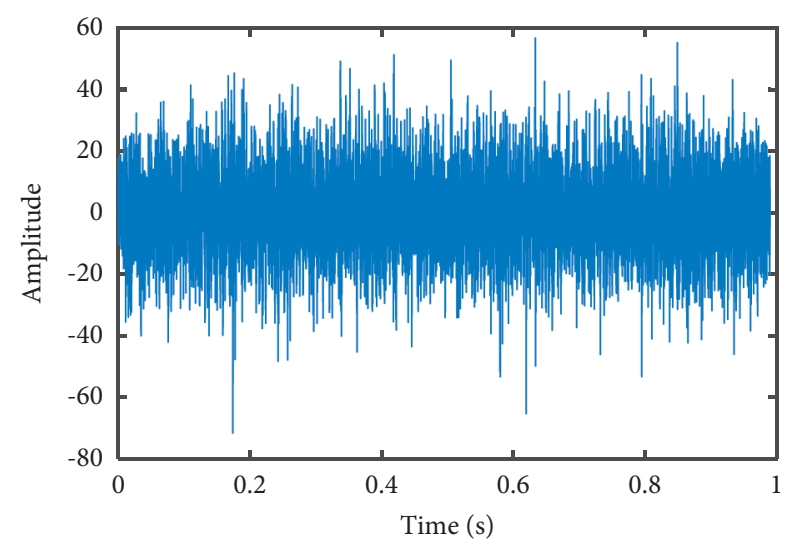

(c)

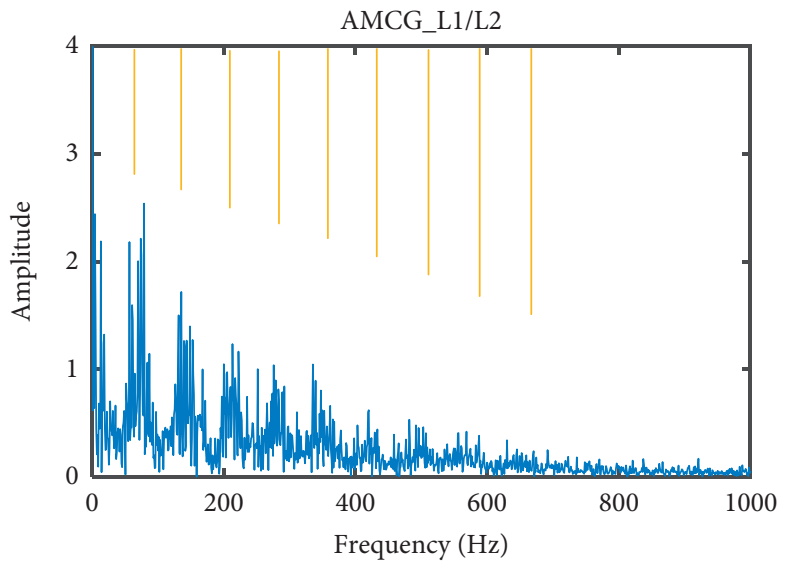

(b)

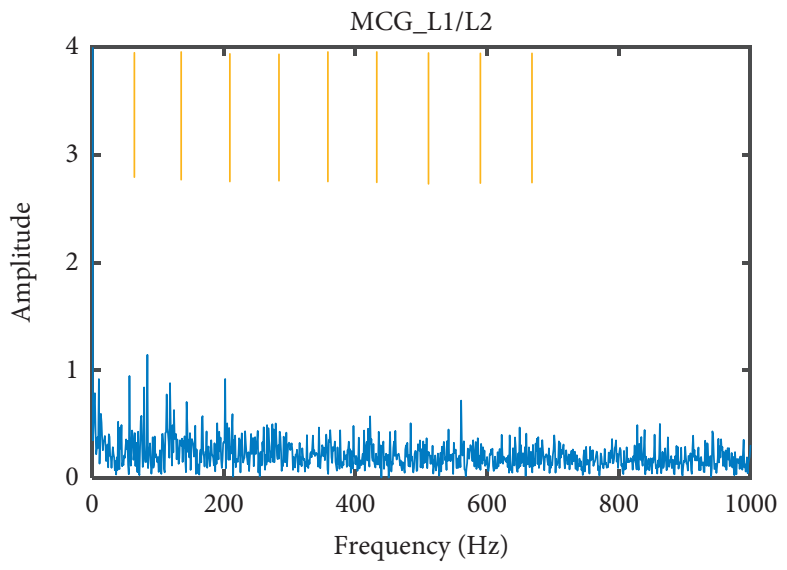

(d)

FIgUre 21: Continued. 


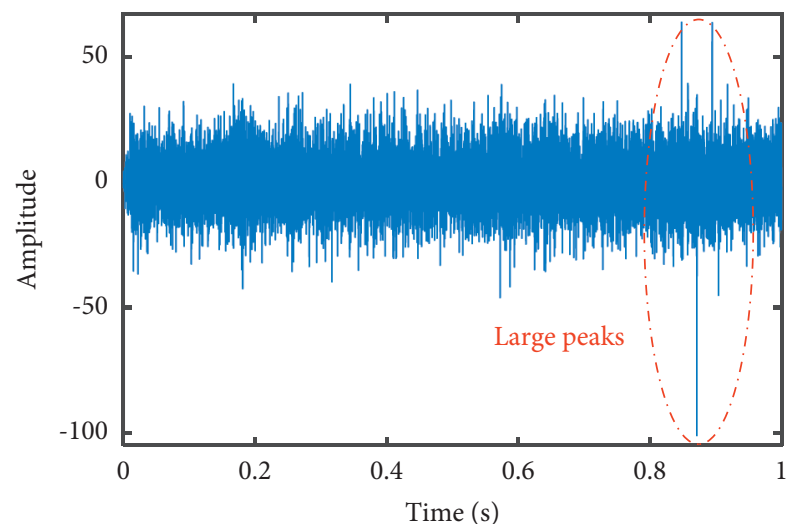

(e)

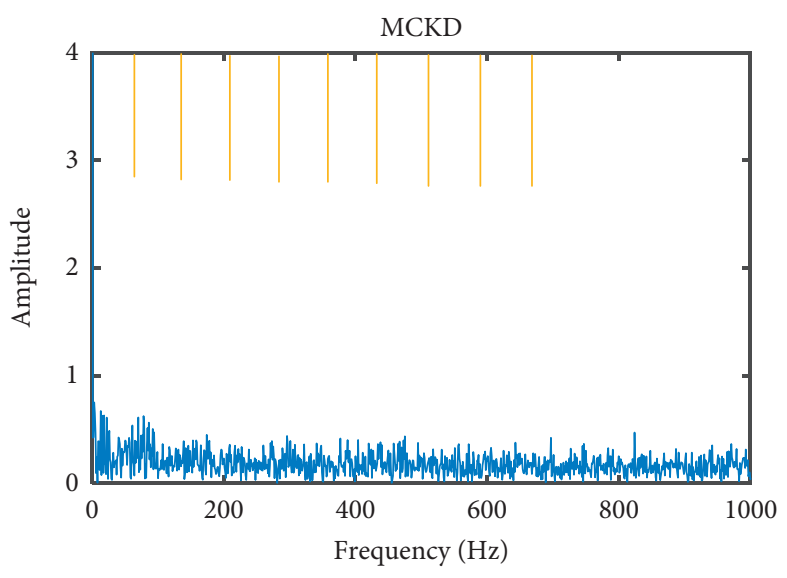

(f)

Figure 21: Processing results of AMCG- $L p / L q-\mathrm{D}, \mathrm{MCG}-L p / L q-\mathrm{D}$, and MCKD for concurrent fault experimental vibration signal (with the input of $71 \mathrm{~Hz}$ ): (a), (c), and (e) waveforms obtained by AMCG- $L p / L q-D$, MCG- $L p / L q-D$, and MCKD; (b), (d), and (f) corresponding envelope spectra.

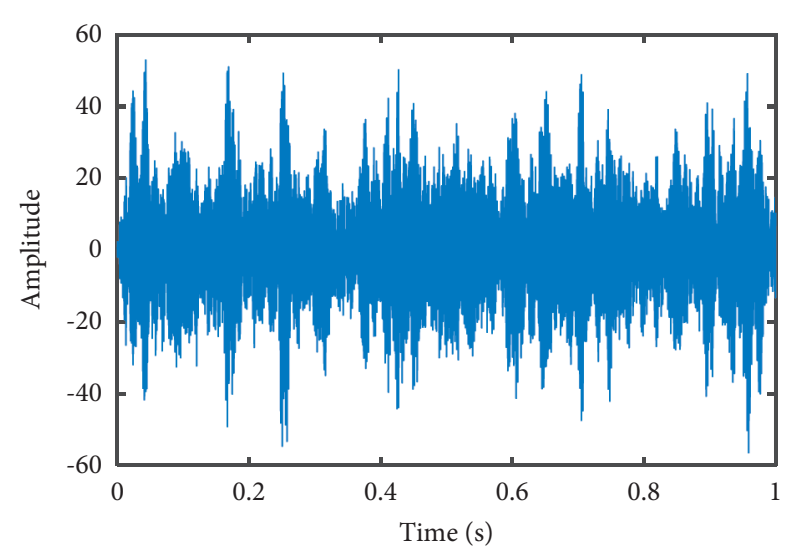

(a)

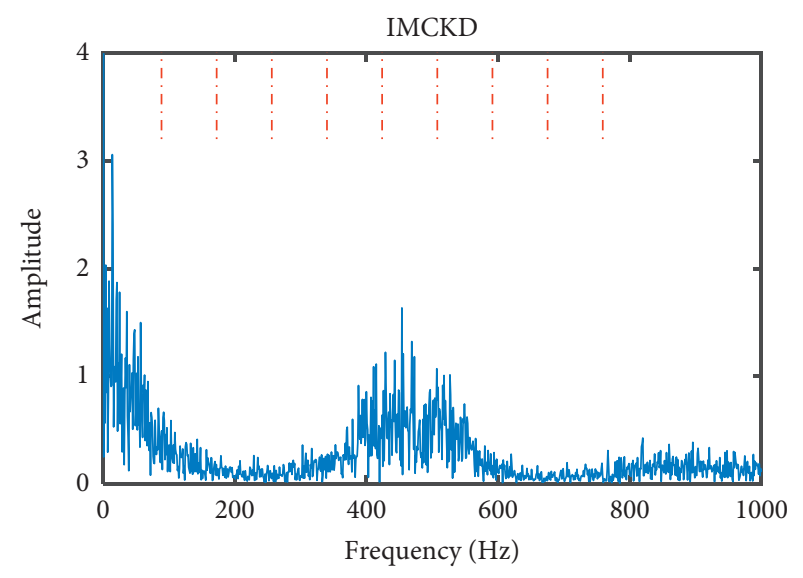

(b)

FIGURE 22: Processing results of IMCKD for concurrent fault experimental vibration signal: (a) waveform; (b) envelope spectrum.

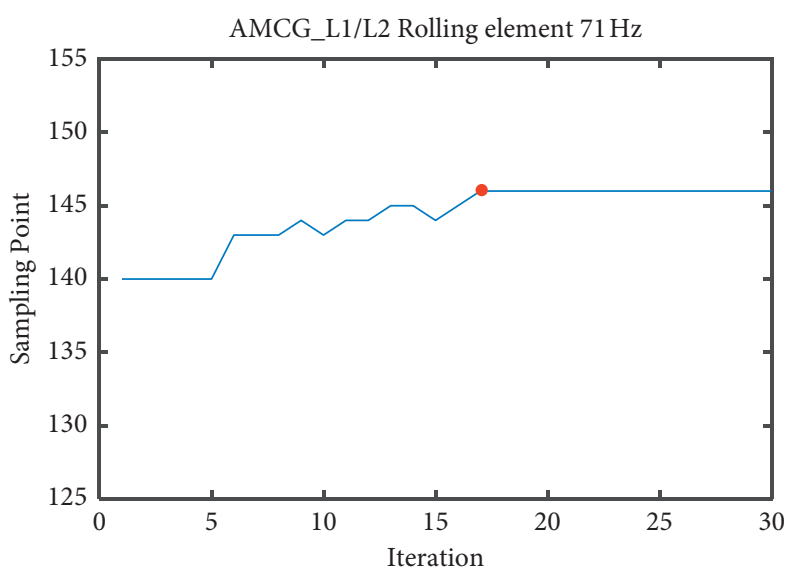

(a)

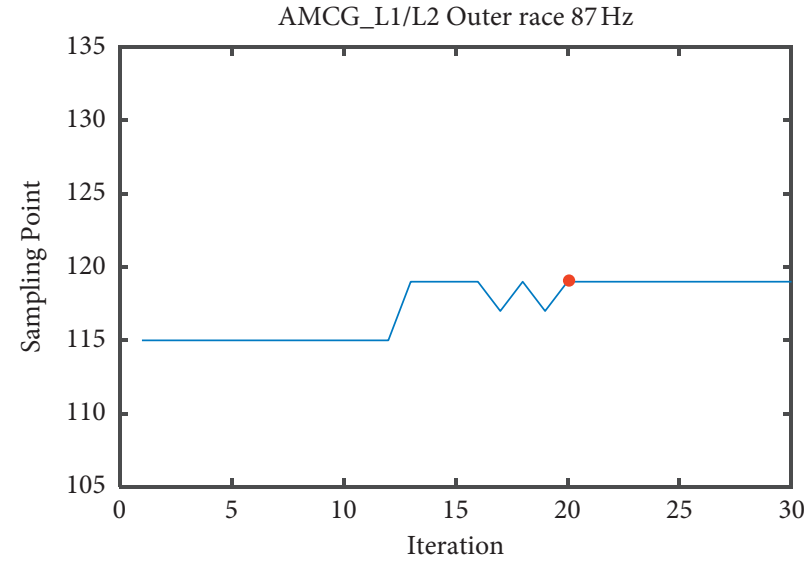

(b)

Figure 23: Continued. 


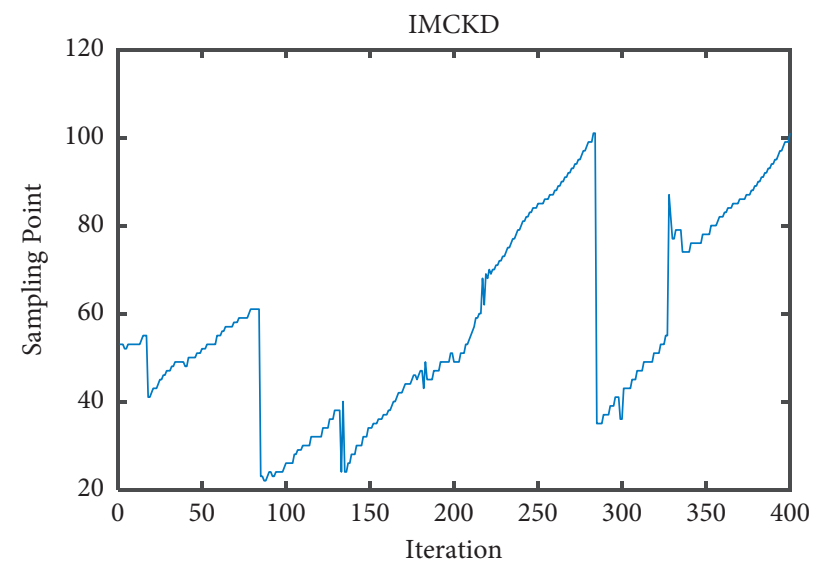

(c)

FIGURE 23: Period estimation of AMCG- $L p / L q$-D and IMCKD for concurrent fault experimental vibration signal: (a) AMCG- $L p / L q$-D with the input of $71 \mathrm{~Hz}$ (to detect the rolling element fault of $67.86 \mathrm{~Hz}$ ); (b) AMCG- $L p / L q$-D with the input of $87 \mathrm{~Hz}$ (to detect the outer-race fault of $83.33 \mathrm{~Hz}$ ); (c) IMCKD.

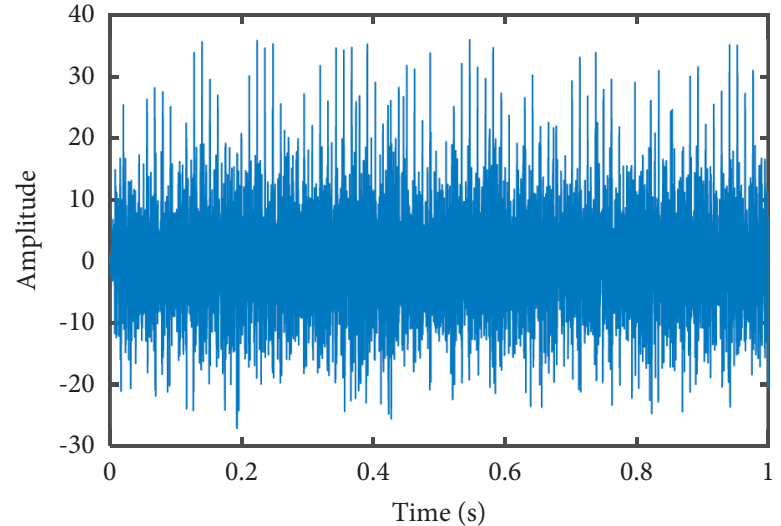

(a)

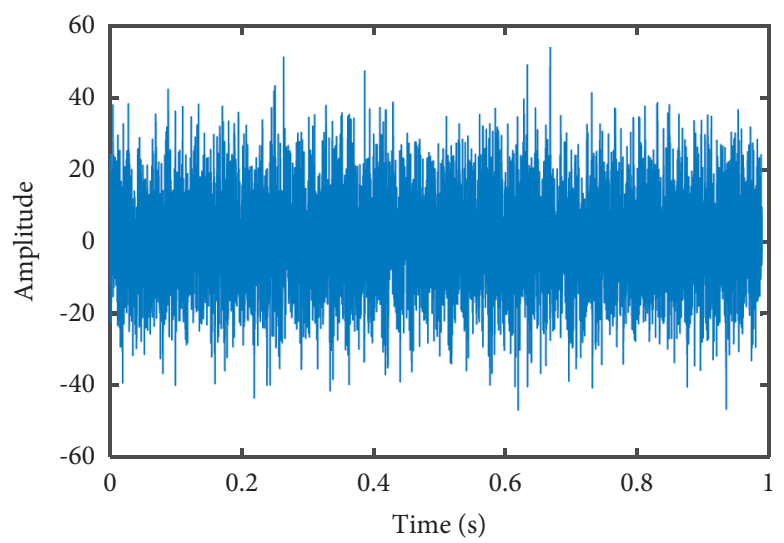

(c)

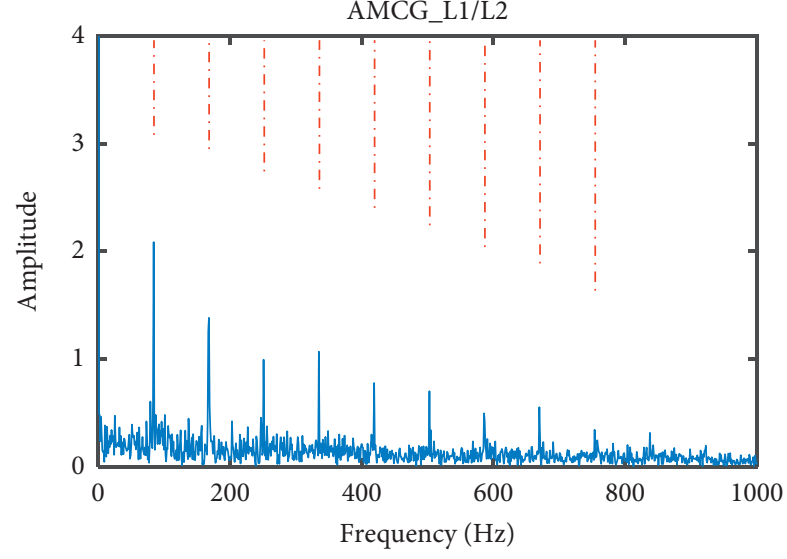

(b)

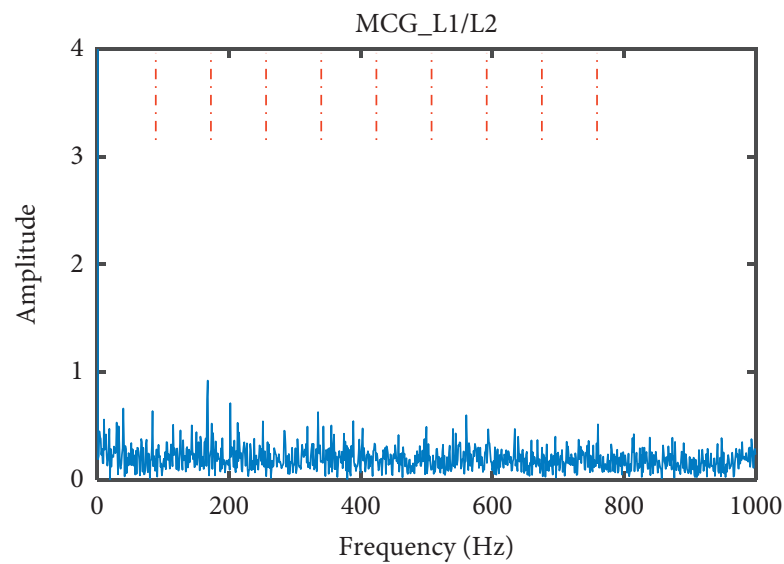

(d)

Figure 24: Continued. 


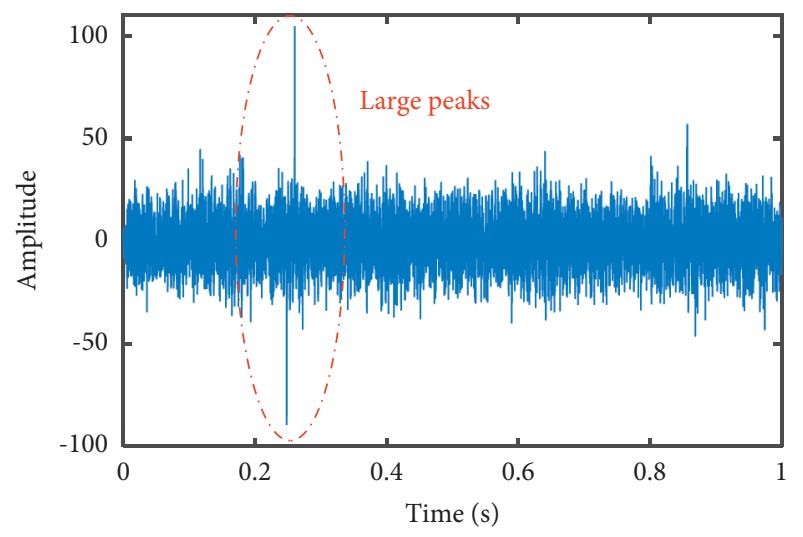

(e)

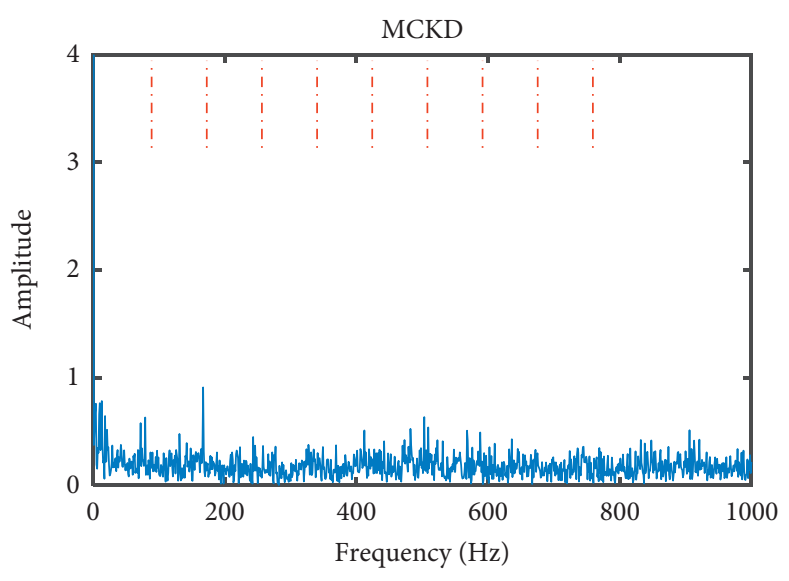

(f)

Figure 24: Processing results of AMCG- $L p / L q-\mathrm{D}$, MCG- $L p / L q-\mathrm{D}$, and MCKD for concurrent fault experimental vibration signal (with the input of $87 \mathrm{~Hz}$ ): (a), (c), and (e) waveforms obtained by AMCG- $L p / L q-\mathrm{D}, \mathrm{MCG}-L p / L q-\mathrm{D}$, and MCKD; (b), (d), and (f) corresponding envelope spectra.

\section{Conclusions}

In this paper, a BD independent of the precise prior fault period is proposed, AMCG- $L p / L q-D$. It can still detect the periodic impulse associated with the fault from the vibration signals with strong noise even when the input prior period is not precise. Therefore, the proposed method can be considered as an improvement to significantly expand the application value of MCG- $L p / L q-D$, because in engineering practice, the fault period obtained through theoretical calculation is often not precise. It overcomes the problem of the dependence of MCG-Lp/ $L q-\mathrm{D}$ on the precise prior period, which allows the proposed method to have a wider range of applications than MCG- $L p / L q-D$. Simulation and experimental case studies verify three advantages of the proposed method. Firstly, the proposed method can gradually estimate the real fault period in iteration without significantly increasing the computational complexity. This ensures that the proposed method will not always use an imprecise fault period to iterate, thus guaranteeing the performance of fault feature extraction for the proposed method, which is unable to be achieved by MCG-Lp/Lq-D. Secondarily, compared with the fully adaptive period estimation technique used by IMCKD, the APEC technique used by AMCG-Lp/Lq-D still has better ability of precise fault period detection even under strong noise interference. Thirdly, the proposed method still has the ability to diagnose concurrent faults. Based on the fault feature detection performance of the proposed method under very low SNR conditions, the proposed method is more suitable for bearing fault diagnosis tasks in engineering practice than MCG-Lp/Lq-D.

\section{Data Availability}

The experimental data are based on the CRRC project and are confidential.

\section{Conflicts of Interest}

The authors declare that they have no conflicts of interest.

\section{Acknowledgments}

This work was supported by the Research Fund of the State Key Laboratory of Traction Power (no. 2020TPL-T14), the National Key R\&D Program of China (nos. 2020YFB1200300ZL-03 and 2018YFB1201603-14), the Fundamental Research Foundations for the Central Universities (no. 2682020CX50), the China Postdoctoral Science Foundation (no. 2019M663899XB), and the National Natural Science Foundation of China (no. 51905453).

\section{References}

[1] J. Antoni, "The spectral kurtosis: a useful tool for characterising non-stationary signals," Mechanical Systems and Signal Processing, vol. 20, no. 2, pp. 282-307, 2006.

[2] J. Antoni and R. B. Randall, "The spectral kurtosis: application to the vibratory surveillance and diagnostics of rotating machines," Mechanical Systems and Signal Processing, vol. 20, no. 2, pp. 308-331, 2006.

[3] J. Antoni, "Fast computation of the kurtogram for the detection of transient faults," Mechanical Systems and Signal Processing, vol. 21, no. 1, pp. 108-124, 2007.

[4] Q. Zhou, Y. Zhang, C. Yi, J. Lin, L. He, and Q. Hu, "Convolutional sparse coding using pathfinder algorithm-optimized orthogonal matching pursuit with asymmetric Gaussian chirplet model in bearing fault detection," IEEE Sensors Journal, vol. 21, no. 16, pp. 18132-18145, 2021.

[5] Z. Xing, C. Yi, J. Lin, and Q. Zhou, "Multi-component fault diagnosis of wheelset-bearing using shift-invariant impulsive dictionary matching pursuit and sparrow search algorithm," Measurement, vol. 178, Article ID 109375, 2021.

[6] Z. Xing, J. Lin, Y. Huang, and C. Yi, "A feature extraction method of wheelset-bearing fault based on wavelet sparse representation with adaptive local iterative filtering," Shock and Vibration, vol. 2020, Article ID 2019821, 20 pages, 2020. 
[7] J. Antoni, "Cyclic spectral analysis of rolling-element bearing signals facts and fictions," Journal of Sound and Vibration, vol. 304, pp. 497-529, 2007.

[8] M. Buzzoni, J. Antoni, and G. D’Elia, “Blind deconvolution based on cyclostationarity maximization and its application to fault identification," Journal of Sound and Vibration, vol. 432, pp. 569-601, 2018.

[9] A. Raad, J. Antoni, and M. Sidahmed, "Indicators of cyclostationarity: theory and application to gear fault monitoring," Mechanical Systems and Signal Processing, vol. 22, no. 3, pp. 574-587, 2008.

[10] J. Antoni, F. Bonnardot, A. Raad, and M. El Badaoui, "Cyclostationary modelling of rotating machine vibration signals," Mechanical Systems and Signal Processing, vol. 18, no. 6, pp. 1285-1314, 2004.

[11] Z. Wang, N. Yang, N. Li, W. Du, and J. Wang, "A new fault diagnosis method based on adaptive spectrum mode extraction," Structural Health Monitoring, vol. 20, no. 6, pp. 3354-3370, 2021.

[12] Y. Miao, M. Zhao, Y. Yi, and J. Lin, "Application of sparsityoriented VMD for gearbox fault diagnosis based on built-in encoder information," ISA Transactions, vol. 99, pp. 496-504, 2020.

[13] Y. Miao, M. Zhao, V. Makis, and J. Lin, "Optimal swarm decomposition with whale optimization algorithm for weak feature extraction from multicomponent modulation signal," Mechanical Systems and Signal Processing, vol. 122, pp. 673691, 2019.

[14] Z. Wang, X. He, B. Yang, and N. Li, "Subdomain adaptation transfer learning network for fault diagnosis of roller bearings," IEEE Transactions on Industrial Electronics, p. 1, 2021.

[15] Z. Wang, W. Zhao, W. Du, N. Li, and J. Wang, "Data-driven fault diagnosis method based on the conversion of erosion operation signals into images and convolutional neural network," Process Safety and Environmental Protection, vol. 149, pp. 591-601, 2021.

[16] R. A. Wiggins, "Minimum entropy deconvolution," Geoexploration, vol. 16, no. 1-2, pp. 21-35, 1978.

[17] W. Gray, Variable norm deconvolution, Ph.D. thesis, Stanford University, Stanford, CA, USA, 1979.

[18] X. Jia, M. Zhao, Y. Di, P. Li, and J. Lee, "Sparse filtering with the generalized $l p / l q$ norm and its applications to the condition monitoring of rotating machinery," Mechanical Systems and Signal Processing, vol. 102, pp. 198-213, 2018.

[19] Z. Wang, J. Zhou, W. Du, Y. Lei, and J. Wang, "Bearing fault diagnosis method based on adaptive maximum cyclostationarity blind deconvolution," Mechanical Systems and Signal Processing, vol. 162, Article ID 108018, 2022.

[20] J.-Y. Lee and A. K. Nandi, "Extraction OF impacting signals using blind deconvolution," Journal of Sound and Vibration, vol. 232, no. 5, pp. 945-962, 2000.

[21] N. Sawalhi, R. B. Randall, and H. Endo, "The enhancement of fault detection and diagnosis in rolling element bearings using minimum entropy deconvolution combined with spectral kurtosis," Mechanical Systems and Signal Processing, vol. 21, no. 6, pp. 2616-2633, 2007.

[22] L. He, D. Wang, C. Yi, Q. Zhou, and J. Lin, "Extracting cyclostationarity of repetitive transients from envelope spectrum based on prior-unknown blind deconvolution technique," Signal Processing, vol. 183, Article ID 107997, 2021.

[23] G. L. McDonald and Q. Zhao, "Multipoint optimal minimum entropy deconvolution and convolution fix: application to vibration fault detection," Mechanical Systems and Signal Processing, vol. 82, pp. 461-477, 2017.
[24] G. L. McDonald, Q. Zhao, and M. J. Zuo, "Maximum correlated Kurtosis deconvolution and application on gear tooth chip fault detection," Mechanical Systems and Signal Processing, vol. 33, pp. 237-255, 2012.

[25] X. Jia, M. Zhao, M. Buzza, Y. Di, and J. Lee, “A geometrical investigation on the generalized $\mathrm{lp} / \mathrm{lq}$ norm for blind deconvolution," Signal Processing, vol. 134, pp. 63-69, 2017.

[26] L. He, Y. Li, Y. Liu, and J. Lin, "Minimum correlated generalized $L p / L q$ deconvolution for recovering repetitive impacts from a vibration mixture," IEEE Sensors Journal, vol. 21, pp. 2043-2054, 2021.

[27] Q. Zhou, Y. Zhang, J. Tang, J. Lin, L. He, and C. Yi, “Blind deconvolution technique based on improved correlated generalized $L p / L q$ norm for extracting repetitive transient feature," IEEE Transactions on Instrumentation and Measurement, vol. 70, pp. 1-21, 2021.

[28] J. Antoni and R. B. Randall, "A stochastic model for simulation and diagnostics of rolling element bearings with localized faults," Journal of Vibration and Acoustics, vol. 125, no. 3, pp. 282-289, 2003.

[29] D. Ho and R. B. Randall, "Optimisation OF bearing diagnostic techniques using simulated and actual bearing fault signals," Mechanical Systems and Signal Processing, vol. 14, no. 5, pp. 763-788, 2000.

[30] Y. Miao, M. Zhao, J. Lin, and Y. Lei, "Application of an improved maximum correlated kurtosis deconvolution method for fault diagnosis of rolling element bearings," Mechanical Systems and Signal Processing, vol. 92, pp. 173195, 2017.

[31] Y. Miao, B. Zhang, M. Zhao, and J. Lin, "Period-oriented multi-hierarchy deconvolution and its application for bearing fault diagnosis," ISA Transactions, vol. 114, pp. 455-469, 2021.

[32] B. Chen, W. Zhang, D. Song, and Y. Cheng, "Blind deconvolution assisted with periodicity detection techniques and its application to bearing fault feature enhancement," Measurement, vol. 159, Article ID 107804, 2020.

[33] M. Zhao and X. Jia, "A novel strategy for signal denoising using reweighted SVD and its applications to weak fault feature enhancement of rotating machinery," Mechanical Systems and Signal Processing, vol. 94, pp. 129-147, 2017.

[34] C. Ding, M. Zhao, J. Lin, and J. Jiao, "Multi-objective iterative optimization algorithm based optimal wavelet filter selection for multi-fault diagnosis of rolling element bearings," ISA Transactions, vol. 88, pp. 199-215, 2019.

[35] B. Zhang, Y. Miao, J. Lin, and Y. Yi, "Adaptive maximum second-order cyclostationarity blind deconvolution and its application for locomotive bearing fault diagnosis," $\mathrm{Me}$ chanical Systems and Signal Processing, vol. 158, Article ID 107736, 2021.

[36] P. D. McFadden and J. D. Smith, "Model for the vibration produced by a single point defect in a rolling element bearing," Journal of Sound and Vibration, vol. 96, no. 1, pp. 69-82, 1984.

[37] Y. Huang, J. Lin, Z. Liu, and C. Huang, "A morphological filtering method based on particle swarm optimization for railway vehicle bearing fault diagnosis," Shock and Vibration, vol. 2019, Article ID 2593973, 16 pages, 2019.

[38] J. Ngiam, P. W. Koh, Z. Chen, S. Bhaskar, and A. Y. Ng, "Sparse filtering," in Proceedings of the Conference and Workshop on Neural Information Processing Systems NIPS, pp. 1125-1133, Granada Spain, December 2011.

[39] R. B. Randall and J. Antoni, "Rolling element bearing diagnostics-A tutorial," Mechanical Systems and Signal Processing, vol. 25, no. 2, pp. 485-520, 2011. 
[40] X. Xu, "Envelope harmonic-to-noise ratio for periodic impulses detection and its application to bearing diagnosis," Measurement, vol. 13, 2016.

[41] M. Zhao, J. Lin, Y. Miao, and X. Xu, "Detection and recovery of fault impulses via improved harmonic product spectrum and its application in defect size estimation of train bearings," Measurement, vol. 91, pp. 421-439, 2016.

[42] J. Bard, A Review of: Engineering Optimization: Theory and Practice, Wiley and Sons, Hoboken, NJ, USA, 1996. 